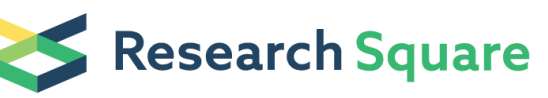 \\ Preprints are preliminary reports that have not undergone peer review. \\ They should not be considered conclusive, used to inform clinical practice, \\ or referenced by the media as validated information.
}

\section{Feruloyl Esterase Fae1 is Required Specifically for Host Colonisation by The Rice-Blast Fungus Magnaporthe Oryzae}

\section{Akhil Thaker}

The Maharaja Sayajirao University of Baroda Faculty of Science

Khyati Mehta

The Maharaja Sayajirao University of Baroda Faculty of Science

Rajesh Patkar ( $\square$ rajeshpatkar@iitb.ac.in )

Indian Institute of Technology Bombay https://orcid.org/0000-0001-7266-2394

\section{Research Article}

Keywords: Ferulic acid, Blast disease, Magnaporthe, Host invasion, Cell wall degradation

Posted Date: June 18th, 2021

DOI: https://doi.org/10.21203/rs.3.rs-591209/v1

License: (c) (i) This work is licensed under a Creative Commons Attribution 4.0 International License.

Read Full License

Version of Record: A version of this preprint was published at Current Genetics on September 15th, 2021. See the published version at https://doi.org/10.1007/s00294-021-01213-z. 
Feruloyl esterase Fae1 is required specifically for host colonisation by the rice-blast fungus

\section{Magnaporthe oryzae}

Akhil Thaker ${ }^{1}$, Khyati Mehta $^{1}$, Rajesh Patkar ${ }^{1, \#, *}$

${ }^{1}$ Bharat Chattoo Genome Research Centre, Department of Microbiology and Biotechnology

Centre, The Maharaja Sayajirao University of Baroda, Vadodara, Gujarat, India 390002

\# Present address: Department of Biosciences and Bioengineering, Indian Institute of

Technology Bombay, Powai, Mumbai, Maharashtra, India 400076

*Author for correspondence - Rajesh Patkar - rajeshpatkar@iitb.ac.in

Key words: Ferulic acid; Blast disease; Magnaporthe; Host invasion; Cell wall degradation 
$3 \quad$ Plant cell wall acts as a primary barrier for microbial pathogens during infection. A cell wall

4 degrading enzyme thus may be a crucial virulence factor, as it may aid the pathogen in

5 successful host invasion. Nine genes coding for feruloyl esterases (Fae), likely involved in plant

6 cell wall degradation, have been annotated in the genome of the cereal-blast fungus

7 Magnaporthe oryzae. However, role of any Fae in pathogenicity of M. oryzae remains hitherto

8 under explored. Here, we identified FAE1 gene (MGG_08737) that was significantly upregulated

9 during host penetration and subsequent colonisation stages of infection. Accordingly, while

10 deletion of FAE1 in M. oryzae did not affect the vegetative growth and asexual development, the

11 fael $\Delta$ mutant showed significantly reduced pathogenesis on rice plants, mainly due to impaired

12 host invasion and colonisation. Very few $(<10 \%)$ fae $1 \Delta$ appressoria that formed the primary

13 invasive hyphae, failed to elaborate from the first invaded cell to the neighboring plant cells.

14 Interestingly, exogenously added glucose, as a simple carbon source, or ferulic acid, a product of

15 the Fae activity, significantly supported the invasive growth of the fae $1 \Delta$ mutant. We show that

16 the Fae1-based feruloyl esterase activity, by targeting the plant cell wall, plays an important role

17 in accumulating ferulic acid and/or sugar molecules, as a likely energy source, to enable host

18 invasion and colonisation by M. oryzae. Given its role in plant cell wall digestion and host

19 colonisation, M. oryzae Fael could be a potential candidate for a novel antifungal strategy and a

20 biotechnological application in biofuel production. 
Microbial phytopathogens encounter plant cell wall as a major obstruction while invading and successfully colonizing the host. Typical plant cell wall is primarily composed of three polysaccharides namely cellulose (microfibrils), hemicellulose (xylan and xylan derivatives) and pectin, interconnected via ferulic acid bridges to form a rigid mesh-like structure (Harris and Hartley, 1977; Bunzel et al., 2001). This mesh-like network is required for providing strength and integrity to the plant cell wall. Phytopathogens, such as fungi and bacteria, deploy different ways to overcome this physical cell wall barrier, mainly to get entry into the host cell and colonise the tissue for nutrient acquisition. While bacterial pathogens prefer passive host entry via hydathodes or stomatal openings, fungal pathogens have evolved mechanisms to breach the primary barrier of plant hosts. The necrotrophic fungal pathogens use cell wall degrading enzymes (CWDE) to invade/colonise the plant tissue (Cosgrove, 2001); whereas the biotrophic or hemibiotrophic fungal pathogens, in addition to CWDE, depend on specialised host entry enabled by infection structures called as appressorium (Howard et al., 1991). While, CWDEs are fairly studied with respect to virulence of phytopathogenic fungi, they are also implemented in industrial applications such as processing of plant cell walls for efficient production of biofuels. Ever-increasing collection of genome sequences reveals that CWDEs offer a wide diversity, both in terms of number and expansion of gene families, across phytopathogenic fungi (Kubicek et al., 2014). It is therefore important to study different CWDEs, which may lead to identification of novel virulence determinants in different phytopathogenic fungi.

Feruloyl esterases (Ferulic acid esterases, Fae; EC 3.1.1.73), a subclass of carboxylic acid esterases (EC 3.1.1.1), also belong to one such group of CWDEs. The Fae hydrolyses the ester bonds in the feruloyl-polysaccharide complex in the plant cell wall and thereby releases ferulic acid and polysaccharide (Faulds and Williamson, 1994; de Vries et al., 1997). 
Breakage of these ester bonds leads to loss of elasticity and plasticity and subsequent weakening of the plant cell wall. Feruloyl esterases have been classified into four classes type A, B, C and D - depending upon sequence attributes and ability to act on wide range of substrates (Crepin et al., 2004). Crystal structure of feruloyl esterase from Aspergillus niger (AnFaeA) has revealed that it is a modular enzyme with a catalytic and a non-catalytic cellulose-binding domain (Hermoso et al., 2004). Involvement of fungal feruloyl esterases with differential activity during plant infection have been studied in different pathosystems. In Fusarium graminearum, feruloyl esterases, particularly FaeB1 and FaeD1, are found to be upregulated during infection or in response to aromatic compounds such as ferulic acid, caffeic acid and p-coumaric acid and also in the presence of carbon sources such as xylose, glucose and galactose (Balcerzak et al., 2012). However, FaeB1 and FaeD1 are not required for pathogenicity on wheat (Balcerzak et al., 2012). On the other hand, expression of Fae is not only upregulated during infection but also plays an essential role in pathogenesis in the apple tree canker pathogen Valsa mali (Xu et al., 2017).

Magnaporthe oryzae, a hemibiotrophic phytopathogenic filamentous fungus, causes blast disease in rice and other important cereal crops worldwide (Valent and Khang, 2010). Over several years, rice-blast disease has been widely used as a model pathosystem to study plantpathogen interactions (Ebbole, 2007; Patkar et al., 2015). The infection cycle of M. oryzae starts with conidial germination in the presence of moisture, followed by perception of specific cues from the host surface, leading to development of appressorium. The enormous turgor pressure generated inside the appressorium helps the fungus in penetrating the host cell. While the intracellular turgor pressure inside the appressorium contributes in generating the mechanical force, the localised loosening of the host cell wall underneath the appressorium is important and carried out by certain plant cell wall digesting enzymes secreted by the fungal pathogen. During subsequent host tissue invasion, the penetration peg 
differentiates into bulbous primary invasive hypha that elaborates within the first invaded cell. Once inside the first host cell, the fungal pathogen uses different strategies to evade plant immunity to colonise the tissue and for disease progression (Skamnioti and Gurr, 2008; Patkar et al., 2015).

Role of CWDEs in host penetration and thereby virulence has been studied in M. oryzae. Endo-xylanases and cellulases are significantly upregulated during plant infection and required for penetration and virulence in M. oryzae (Nguyen et al., 2011; Vu et al., 2012). Further, a secreted feruloyl esterase A, encoded by MGG_01403.5, in M. oryzae is found to be expressed during post-penetration stage (72 hpi onwards) of rice infection, but does not play a significant role in pathogenesis of the fungus (Zheng et al., 2009). Interestingly, the FAE gene family in M. oryzae is relatively expanded when compared to that in nonpathogenic counterparts such as Neurospora crassa and Aspergillus nidulans, which have only one and three $F A E$ genes, respectively (Dean et al., 2005). Hitherto, role of any other FAEs has not been studied in M. oryzae. Here, we studied the expression profiles of nine FAE genes, to identify an early invasion-related Fae function. We show a crucial role for one of the Fae, in host invasion and tissue colonisation. Our in silico analysis of these Fae also highlights a likely diversification of the sequences in a host-specific manner.

\section{Experimental Procedures}

\section{Fungal culture and growth conditions}

M. oryzae wild-type (WT) B157 strain (MTCC accession no. 12236; Kachroo et al., 1994) belonging to the international race IC9 was used in this study. Fungus was grown and maintained on Prune Agar (PA) plates as described earlier (Soundararajan et al., 2004). 
94 Vegetative growth of the fungus on PA plates was allowed for 10 days at $28{ }^{\circ} \mathrm{C}$, with initial 3 days incubation under dark conditions followed by 7 days incubation under constant illumination for conidiation. Vegetative growth was assessed by visual observation of the colony morphology and by measuring the colony diameter. Conidia were harvested as described previously (Patkar et al., 2010), followed by microscopic observation of the conidial morphology. Harvested conidia were counted using a hemocytometer and reported in terms of total number of conidia per unit area of the colony.

Assay for appressorial development was performed by spotting $20 \mu \mathrm{L}$ conidial suspension $\left(\sim 10^{4}\right.$ conidia/mL) on an inductive (hydrophobic) cover glass (22 mm, no. 1; Microcil Ltd., India) for up to 24 hours at $25{ }^{\circ} \mathrm{C}$ under humid conditions, followed by assessment of appressorium formation by microscopic observation.

Nucleic acids and proteins were isolated by grinding in liquid nitrogen the fungal biomass obtained from vegetative culture grown in an appropriate liquid medium for $2-3$ days at 28 ${ }^{\circ} \mathrm{C}$, followed by the standard protocols mentioned earlier (Dellaporta et al., 1983; Kachroo et al., 1997).

\section{Identification and in silico analysis of fungal $F A E S$}

Initial identification of putative feruloyl esterases in $M$. oryzae was done using NCBI protein BLAST (https://blast.ncbi.nlm.nih.gov/Blast.cgi?PAGE=Proteins) with known Fae sequences from Aspergillus oryzae (AoFaeB; PDB: 3WMT_B) and Neurospora crassa (NcFaeB; GenBank: AJ293029). Multiple sequence alignment of these putative Fae was carried out using ClustalW feature in MEGA tool to check the presence of GXSXG conserved motif (Dilokpimol et al., 2016). Presence of the characteristic $\alpha / \beta$ hydrolase domain was checked 
using the NCBI conserved domain database

(http://www.ncbi.nlm.nih.gov/Structure/cdd/wrpsb.cgi; Marchler-Bauer et al., 2015). Further, percentage identity among all these $M$. oryzae Fae was checked by performing multiple sequence alignment of protein sequences using Clustal Omega (Sievers et al., 2011; Sievers and Higgins, 2018), followed by plotting the distance matrix heatmap using R tool (Supplementary Fig. S5).

Phylogenetic analysis of Fae in different host-specific isolates of M. oryzae (GY11, P131, Y34, PH14, US71, MZ5-1-6, CD156, BR32) was carried out using the annotated protein sequences, available in the NCBI and GEMO (http://genome.jouy.inra.fr/gemo/) databases, from these isolates. Protein sequences of putative Fae in M. oryzae 70-15 strain was used as a query to perform BlastP analysis with a custom database containing all the protein sequences from the aforementioned different isolates. Phylogenetic analyses were carried out with MEGA7 (Kumar et al., 2016), using the Maximum Likelihood method based on the JTT matrix-based model.

For phylogenetic analysis of feruloyl esterases across different fungal taxa, genome sequences of 21 representative fungal species (Table S1) were retrieved from the NCBI database. Proteomes were mined for the presence of the conserved domain Tannase (Pfam ID: PF07519) or Esterase_PHB (Pfam ID: PF10503), using hmm-search (hidden Markov model-search) of HMMER suite (parameters -cut_tc; Hancock \& Bishop, 2004). Poorly aligned sequences were removed using TrimAl (Capella-Gutierrez et al., 2009; parameters automated1). Alignment of Tannase domain containing sequences was used to construct the maximum likelihood phylogenetic tree, using IQ-TREE (Nguyen et al., 2015). Firstly, best fit model (LG+I+G4) was chosen based on the Bayesian Information criterion (BIC), followed by assessment of phylogenetic tree for branch support with ultrafast bootstrap (parameters -m 
model -alrt 1000 -bb 1000 -nt AUTO). Phylogenetic tree was visualized using iTOL (Letunic and Bork, 2019).

\section{Signal peptide prediction and validation}

Prediction of conventional secretory signal peptide was carried out using SignalP 4.0 tool (http://www.cbs.dtu.dk/services/SignalP-4.0/; Petersen et al., 2011). Presence of functional signal peptide was confirmed for two randomly selected FAEs - MGG_05529 and MGG_07294 - using Yeast Secretion Trap (YST) approach as described (Lee et al., 2006). The ORFs of MGG_05529 and MGG_07294 were PCR-amplified from M. oryzae WT genomic DNA, using the following primer pairs (MGG_05529-F: 5'ATGGACTCGTCAATCATTCACTGG-3' and MGG_05529-R: 5'CCCCATTCCACTTTGACCTG-3'; MGG_07294-F: 5'-ATGCGTTTCTCCAGCATCTTC3' and MGG_07294-R: 5'-CGCAATGAGACCAAAGAACC-3'). Individual PCR products were subjected to blunt end cloning in pYST2 vector at NotI site after end-filling, followed by E. coli $\mathrm{DH} 5 \alpha$ transformation. Transformants obtained on Luria-agar plates containing 100 $\mu \mathrm{g} / \mathrm{mL}$ ampicillin antibiotic were screened by RE digestion and those with desired restriction digestion pattern were confirmed by DNA sequencing. These recombinant plasmids from desired clones of $E$. coli DH5 $\alpha$ were used for yeast (Saccharomyces cerevisiae) DBY $\alpha 2445$ (MAT $\alpha$, suc2 $2-9$, lys2-801, ura3-52, ade2-101) transformation as described previously (Gietz and Woods, 2002). Selected transformants were screened and confirmed by colony PCR. Confirmed S. cerevisiae transformants were spotted on SD (YNB with $\left(\mathrm{NH}_{4}\right)_{2} \mathrm{SO}_{4}$, Lysine, Uracil and Adenine) + sucrose agar selection plates and incubated for 6 days at 28 ${ }^{\circ} \mathrm{C}$. 


\section{Determination of fungal Fae activity}

166 In order to check the effect of host-extract on Fae enzyme activity, 3-day-old vegetative culture of WT M. oryzae grown in liquid YEG (0.2\% Yeast-extract and $1 \%$ glucose $)$ medium with or without crude rice leaf extract was used. Protein samples were prepared from fungal biomass (intracellular proteins) and culture supernatant (secretory proteins), essentially as described earlier (Kachroo et al., 1997), followed by biochemical spectrophotometric enzyme assay using Fae-specific substrate, 4-nitrophenyl ferulate (Institute of Chemistry, Slovak Academy of Sciences, Bratislava), as described previously (Mastihuba et al., 2002). Protein estimation was carried out using Bradford reagent and standard curve method (B6916; Sigma-aldrich, USA). Finally, specific activity $\left(\mathrm{mU} \mathrm{mg}^{-1}\right)$ of Fae was calculated for intracellular and secretory protein fractions from both control and rice-extract-treated samples.

\section{Gene expression analysis by qRT-PCR}

179 For gene expression analysis under host- or pathogenicity-mimic conditions, 3-day old vegetative culture of WT M. oryzae grown in liquid complete medium (CM; 0.5\% Peptone, $0.1 \%$ Yeast-extract, $0.1 \%$ CAA, $0.05 \% \mathrm{KCl}, 0.05 \% \mathrm{MgSO}_{4}, 0.15 \% \mathrm{KH}_{2} \mathrm{PO}_{4}, 1 \%$ glucose, $\left.0.6 \% \mathrm{NaNO}_{3} ; \mathrm{pH} 6.5\right)$ was used. Fungal biomass was harvested and washed thrice with sterile distilled water followed by aseptic transfer in nearly equal amounts to the following seven different media conditions: 1) Minimal medium (MM; $0.05 \% \mathrm{KCl}, 0.05 \% \mathrm{MgSO}_{4}$, $0.15 \% \mathrm{KH}_{2} \mathrm{PO}_{4}, 1 \%$ glucose, $0.6 \% \mathrm{NaNO}_{3} ; \mathrm{pH} 6.5$ ) as a control condition, 2) $\mathrm{MM}$ (w/o glucose $)+0.03 \%$ cutin monomers $(1,16$-hexadecanediol; Sigma); 3) MM (w/o glucose $)+$ $0.03 \%$ ferulic acid (Sigma); 4) $\mathrm{MM}$ minus $\mathrm{NaNO}_{3}$ (nitrogen starvation); 5) $\mathrm{MM}$ (w/o glucose $)+1 \%$ pectin $($ Sigma $) ; 6) \mathrm{MM}($ w/o glucose $)+1 \%$ N-acetylglucosamine (NAG; 
HiMedia) and 7) MM (w/o glucose $)+1 \%$ xylan (Sigma). Fungal cultures were then treated in aforementioned media conditions for 48 hours under shaking conditions at $28{ }^{\circ} \mathrm{C}$, followed by qRT-PCR analysis.

Similarly, FAEs gene expression profile during different stages of infection (in vivo) was studied using a method, with modifications, reported earlier (Skamnioti and Gurr, 2007). Briefly, a 15-20 $\mu \mathrm{L}$ WT conidial suspension $\left(\sim 10^{5}\right.$ conidia/mL; containing $0.05 \%$ gelatin $)$ was drop-inoculated on the surface-sterilized 2 - 3-week old barley leaf blades placed on to kinetin-agar plates, followed by incubation under dark $(8 \mathrm{~h})$ and light $(14 \mathrm{~h})$ cycles at $25{ }^{\circ} \mathrm{C}$. Samples for RNA extraction were collected by excising inoculated portion of the leaf blades at different time-points viz. 12, 24, 48, 72- and 96-hours post inoculation (hpi) along with 3day old vegetative mycelia, grown in liquid CM, as a control condition for qRT-PCR analysis. A sample from mock-inoculation i.e., leaves inoculated with only $0.05 \%$ gelatin solution, was used as a negative control for any non-specific amplification during qRT-PCR.

Total RNA was extracted from the samples collected for each of the above-mentioned conditions/time-points using TRIzol ${ }^{\circledR}$ reagent (Invitrogen, USA), as per the manufacturer's instructions. A total of $2 \mu \mathrm{g}$ of total RNA each was used for the first strand cDNA synthesis using Oligo-(dT) 18 primers (Sigma, India) and M-MuLV reverse transcriptase (New England BioLabs, USA). The first strand cDNA was then subjected to qRT-PCR analysis using a Power SYBR ${ }^{\circledR}$ Green PCR Master Mix (Applied Biosystems, USA), performed on a 7900HT Fast Real-Time PCR System (Applied Biosystems, USA) according to the manufacturer's instructions. Optimized thermal cycling conditions for qRT-PCR were as follows: initial denaturation step at $95{ }^{\circ} \mathrm{C}$ for 15 minutes followed by 40 cycling reactions each at $95{ }^{\circ} \mathrm{C}$ (denaturation step) for 15 seconds and $66^{\circ} \mathrm{C}$ (annealing and extension step) for 45 seconds. Transcript levels in each of the test conditions were calculated by $2^{-\Delta \Delta \mathrm{Ct}}$ method (Livak method; Livak and Schmittgen, 2001) relative to that of the vegetative mycelia grown in 
liquid MM or CM, normalized against $\beta$-tubulin (MGG_00604) transcript levels as an endogenous (loading) control. Primers used for qRT-PCR analysis are listed in the Table S2.

\section{Generation of $F A E 1$ deletion mutant and genetic complementation of fae1 $\triangle$ strain}

FAE1 gene (Gene ID: MGG_08737) deletion construct was made by double-joint PCR approach (Yu et al., 2004) using hygromycin phosphotransferase (HPT) gene as a selectable marker, followed by targeted gene replacement in WT M. oryzae via homologous recombination (Supplementary Fig. S1A). Here, $\sim 1 \mathrm{~kb}$ each of 5' and 3'-untranslated regions (UTR) of FAE1 gene were amplified from WT genomic DNA and fused with $H P T$ gene cassette, using a recombinant PCR, followed by another round of PCR with nested primers to get a specific amplification product (Table S3). This recombinant PCR product was further purified by Na-acetate/ethanol precipitation and used for polyethylene glycol (PEG)mediated fungal transformation (Prakash et al., 2016), using WT M. oryzae protoplasts. Fungal transformants were selected on YEG agar plates containing $200 \mu \mathrm{g} / \mathrm{mL}$ hygromycin. Transformants growing on the selection medium were further screened by locus-specific PCR, where only one out of a total 72 transformants showed the desired amplified PCR product (Supplementary Fig. S1B). This transformant was further analysed by RT-PCR, wherein, as expected, no transcript was detected when compared with the WT or an ectopic transformant (Supplementary Fig. S1C).

Next, the selected transformant was confirmed for site-specific integration (replacement of the FAE1 ORF with the selectable marker gene) by Southern blot hybridization. Genomic DNA was extracted from the WT and fael $\Delta$ strains and further subjected to restriction enzyme digestion with PstI. The HPT gene, used as a probe, was labeled and DNA detection was performed using Alkphos Direct labeling and detection kit (GE Healthcare Ltd., UK) 
following the manufacturer's instructions. Southern blot hybridization analysis confirmed replacement of the FAE1 ORF in the mutant with a single copy of HPT gene cassette (Supplementary Fig. S1D).

For genetic complementation of fael $1 \Delta$ strain, a full-length genomic sequence of $M$. oryzae FAE1 ORF (MGG_08737) along with 5'-UTR ( 1.5 kb) was amplified by PCR using FAE8737-5'UTR(F) and FAE-8737-NST(R) primers (Table S4). The amplified PCR product was cloned at EcoRI/XbaI sites in pFGL889-ILV2 $2^{\text {SUR }}$ based pFGL1010 vector (Yang and Naqvi, 2014; Addgene plasmid \# 119081). The cloned 5'UTR-FAE1 ORF sequence was confirmed by restriction enzyme digestion of the recombinant plasmid pRPL049. Further, the final sequence for complementation was PCR-amplified from the pRPL049 recombinant plasmid using LB-F1 (5'-TGCGGACGTTTTTAATGTACTG-3') and RB-R1 (5'GAAACGACAATCTGATCCAAGC-3') primers. The amplified PCR product was again confirmed by restriction enzyme digestion and purified by gel extraction (QIAEX ${ }^{\circledR}$ II Kit, Qiagen, Germany), and subsequently introduced into fae $1 \Delta$ strain by protoplast transformation (Prakash et al., 2016). As described previously (Yang and Naqvi, 2014), the transformants were selected on Basal Medium (BM) containing $100 \mu \mathrm{g} / \mathrm{mL}$ chlorimuron ethyl, as only those with $I L V 2^{S U R}-5$ 'UTR-FAE1 integrated at the native $I L V 2$ locus will be able to grow on this selection medium. Chlorimuron ethyl resistant transformant was then confirmed by locus-specific PCR using ILV2-5'UTR (5'- TTGTCATCGTCTGACAGGTC) and FAE-8737-5'UTR-R (Table S4) primers and further analysed by RT-PCR, wherein FAE1 transcript could be detected (Fig S1E and S1F).

\section{Whole-plant infection and in vitro host-invasion assays}


261 Whole-plant infection assay was carried out by spraying $\sim 10^{5}$ conidia/mL in $0.05 \% \mathrm{w} / \mathrm{v}$ 262 gelatin onto $\sim 4$-week old rice plants, followed by incubation at 28 to $30^{\circ} \mathrm{C}$ under humid conditions, initially for $24 \mathrm{~h}$ in the dark and then for 4 to 6 days under $14 \mathrm{~h}$ light and $10 \mathrm{~h}$ dark cycles. Development of disease symptoms was monitored regularly during the entire incubation period and recorded at an appropriate time.

Host invasion by the fungal strains was checked by inoculating $\sim 20 \mu \mathrm{L}$ conidial suspension $\left(\sim 10^{4}\right.$ conidia/mL) on to the leaf sheaths obtained from $3-4$-week old host (rice, wheat or barley) plants, followed by incubation at room temperature $\left(<30^{\circ} \mathrm{C}\right)$ under humid conditions. Host invasion was observed and quantified by scoring at least 100 appressoria each, using bright-field microscopy (Olympus BX51, Japan), for the development of invasive hypha in the plant cell underneath, at 48 or 96 hpi. To check the effect of exogenous supply of glucose $(2 \% \mathrm{w} / \mathrm{v})$, ferulic acid $(100 \mathrm{mM})$ or reduced glutathione $(\mathrm{GSH}, 20 \mathrm{mM})$ on host invasion by the blast fungus, the compound was added to the rice leaf sheaths, already inoculated with conidia, at $22 \mathrm{hpi}$, and the resultant invasive hyphal growth was observed at $\sim 48$ hpi.

\section{Statistical analyses}

Quantitative analyses for fungal Fae enzyme activity, transcript levels, vegetative growth, conidiation, appressorial development and host-invasion ability were carried out with three independent sets and the results are reported as mean \pm standard deviation of mean (SDM).

Statistical significance was determined by using two-tailed $t$-test; and $P$-values less than 0.05 (i.e. $<5 \%$ ) were considered as statistically significant. $P<0.05$ is denoted as $*$ and likewise $P<0.01$ as ** and $P<0.001$ as ***. 


\section{Results}

Identification and in silico analysis of feruloyl esterase (FAE) gene(s) in M. oryzae

We performed a protein BLAST analysis using the known Fae sequences from Aspergillus oryzae (AoFaeB; PDB: 3WMT_

B) and Neurospora crassa (NcFaeB; GenBank: AJ293029) as a query against $M$. oryzae 70-15 reference genome database. We found in $M$. oryzae genome a total of nine putative feruloyl esterases that showed homology with either AoFaeB or NcFaeB. While MGG_08737 and MGG_07294 showed highest (47\% and 75\%) identity to AoFaeB and NcFaeB, respectively (Table S2), MGG_03771 showed similarity to both the reference sequences. Feruloyl esterases belong to the $\alpha / \beta$-hydrolase-fold superfamily and catalyze substrate hydrolysis following the mechanism of serine proteases with a conserved GXSXG motif and Ser-His-Asp/Glu catalytic triad (Dilokpimol et al., 2016). Our multiple sequence alignment of all nine putative Fae in M. oryzae showed the presence of the conserved GXSXG motif (Fig. 1A).

Next, we performed an hmm-based search for the presence of Tannase (Pfam ID: PF07519) or Esterase_PHB (Pfam ID: PF10503) domain, another characteristic of Fae, within the protein sequences of twenty-one representative fungi, including $M$. oryzae (70-15), belonging to seven different taxonomic classes. We found in these fungi a total of 203 putative Fae protein sequences, of which 151 showed presence of Tannase domain, whereas the remaining 52 protein sequences contained an Esterase_PHB domain (Table S1). Here, the M. oryzae genome, unlike in the aforementioned GXSXG motif-based analysis, showed twelve putative Fae sequences, wherein nine contained Tannase and the rest three had Esterase_PHB domain. 
Interestingly, majority of the phytopathogenic fungi such as Fusarium spp., V. mali, Magnaporthe spp., Colletotrichum graminicola and Zymoseptoria tritici showed more than one, Tannase domain containing, highly variable Fae sequences. However, Ustilago maydis, a basiodiomycete causal agent of corn smut disease, showed only one Esterase_PHB and zero Tannase domain containing Fae sequence. Intriguingly, the non-pathogenic filamentous fungi such as $N$. crassa, and particularly Aspergillus spp., also showed more than one putative FAE in their genomes; whereas, fission yeast (S. pombe) or budding yeast (S. cerevisiae) did not show any Fae sequence.

Further, we analysed the phylogenetic relationship of all the putative Fae, containing Tannase domain, in the aforementioned fungal species. The phylogenetic tree showed that Fae sequences had a significant genetic diversity and a discontinuous distribution pattern even among multiple Fae of the same species (Fig. 1B). Out of the nine Tannase domain containing M. oryzae Fae (MGG_03771, MGG_03502, MGG_05366, MGG_02261, MGG_05592, MGG_08737, MGG_09404, MGG_09677 and MGG_09732), MGG_08737 (depicted as filled-star in Fig.1B) seemed to be highly diverged and is grouped into a subclade exclusive to Magnaporthales order.

We then performed, using MEGA tool, a phylogenetic analysis of combined sequences of Fae from a few host-specific strains of the blast fungal pathogen isolated from various cereal crops such as rice, wheat and millet (Fig. 1C and Supplementary Fig. S2). Interestingly, our analysis showed divergence of feruloyl esterase genes, especially MGG_9404 and MGG_08737, in a host-specific manner (Fig. 1C and Supplementary Fig. S2), suggesting a crucial function for these sequences in pathogenesis likely towards distinct hosts.

\section{M. oryzae Fae are induced in the presence of host extract and are secretory in nature}


We presumed that M. oryzae Fae would be required to function outside the cell; and hence studied whether these fungal enzymes were secretory in nature. Firstly, we checked the presence or absence of a secretory signal peptide in the amino acid sequences of the aforementioned nine Fae using SignalP program. We found that a conventional secretory signal peptide was present in seven out of nine Fae in M. oryzae (Table S2). In order to validate this observation, two putative $F A E$ genes were randomly selected and subjected to the Yeast Secretion Trap (YST) system as described earlier (Fig. 2A; Lee et al., 2006). The two FAE genes (MGG_05529 and MGG_07294) were then individually cloned in-frame, in the vector pYST2, with the SUC2 invertase gene without its native signal peptide, and transferred individually to the budding yeast (S. cerevisiae) strain DBY $\alpha 2445$, which does not carry its native $S U C 2$ invertase gene. Thus, neither the untransformed yeast strain nor a transformant with the backbone vector would be able to grow on selection medium containing sucrose as the sole carbon source. However, the yeast transformant expressing the Suc2 fused with Fae would be able to utilize sucrose in the selection medium, only if a signal peptide on Fae aids in secretion of the invertase. Indeed, the yeast transformants harboring the plasmid with Suc2 fused to either of the two $F A E$ genes grew on sucrose agar, confirming the presence of a signal peptide and thereby the secretory nature of Fae enzymes in M. oryzae (Fig. 2B).

Next, to check whether the host tissue had any effect on M. oryzae Fae activity, the WT fungal culture was grown in YEG broth with or without crude rice leaf extract. Both, fungal biomass and culture supernatant, were collected separately and subjected to the in vitro enzyme activity assay. We found that the total enzyme activity was significantly higher (166 $\pm 3.33 \mathrm{mU} \mathrm{mg}^{-1} ; P<0.001$ ) in the extracellular (secretory) fraction of the culture grown in the presence of rice leaf extract, when compared with that $\left(29 \pm 0.69 \mathrm{mU} \mathrm{mg}^{-1}\right)$ without the host extract (Fig. 2C). Importantly, the total enzyme activity in the intracellular fraction was 
less and remained largely unchanged even in the presence of the host leaf extract (Fig. 2C).

These results indicate that most M. oryzae Fae are secretory and that their expression is induced by the host-derived factors suggesting a likely role for the CWDE in blast fungal pathogenesis.

\section{M. oryzae Fae express differentially during pathogenesis}

Given that the CWDEs are expressed under tight regulation (Zheng et al., 2009) and that $M$. oryzae Fae secretion was induced in the presence of rice leaf extract, we studied accumulation of nine $F A E$ transcripts in response to individual host cell wall components. Considering the relatively higher turnover of the plant cell wall components during infection, we grew the vegetative culture in media containing - 1) Ferulic acid, 2) Pectin, 3) Xylan, 4) N-acetyl glucosamine (NAG; fungal cell wall component), 5) Cutin monomer (inducer of appressorial development), 6) Nitrogen starvation (mimic of pathogenic development) or 7) Glucose (control condition). Intriguingly, while there was no obvious pattern in accumulation of any particular transcript in response to the plant cell wall components, majority of the FAEs showed a $\geq 2$-fold increase in expression in the presence of NAG (Fig. 3A). Whereas, those of almost all the FAEs were significantly lowered, either due to $\mathrm{N}_{2}$ starvation or in the presence of xylan (Fig. 3A). Our findings suggest that the expression of M. oryzae FAE could be induced by likely activity of plant or fungal chitinase during the host-pathogen interaction.

Next, we studied the expression of FAEs during both pre- and post-host-invasion. The WT conidia were inoculated on detached barley leaves and samples were harvested at specific time-points signifying different stages of blast fungal infection cycle - pre-invasive appressorial development (12 h), host penetration and colonisation (24-48 h) followed by necrotrophic growth phase (48-96 h). The relative transcript levels of all the nine FAEs were 
estimated by qRT-PCR during aforementioned infection stages and compared with those from the vegetative mycelia grown in liquid complete medium. While, almost all the FAE genes showed differential upregulation at different phases of pathogenic life cycle, remarkably, MGG_08737, hereafter referred as FAE1, showed a significant increase in relative transcript levels ( $\sim 300$-fold) during pre-invasive appressorial development and host penetration stages (12 and 24 hpi) when compared to those of the other FAEs from the vegetative mycelia (Fig. 3B). The FAE1 transcript levels further increased ( 470-fold) during the subsequent host colonisation (48 hpi) and remained at elevated level ( 293 -fold) at 72 hpi, followed by a sharp decline ( 27-fold) at 96 hpi (Fig. 3B). Importantly, the FAE1 transcript profile here is in accordance with the global transcriptome reported earlier in $M$. oryzae (Jeon et al., 2020). Our observations indicate that feruloyl esterases, particularly Fae1, has an important role during pathogenesis in the blast fungal pathogen.

\section{M. oryzae Fae1 function is required specifically for host invasion}

Given the significant upregulation of FAE1 during pre- and post-invasion, we generated a fael $\Delta$ mutant to study its role, if any, in fungal development and pathogenesis. We first studied different phenotypic characteristics, such as vegetative mycelial growth and asexual (conidial) development, of the fael $\Delta$ mutant. The vegetative growth of the fael $\Delta$ mutant, after $10 \mathrm{dpi}$, was comparable to that of the WT, where colony size, morphology and melanization on PA plates was similar in both the strains (Fig. 4A). While, the colony diameter of the WT was $7.37 \pm 0.06 \mathrm{~cm}$ that of the $f a e 1 \Delta$ was $7.17 \pm 0.06 \mathrm{~cm}$ (Fig. 4B). Further, total conidiation was determined by harvesting asexual conidia from vegetative culture on PA plates at 10 dpi. The fae $1 \Delta$ produced $102.2 \pm 12.2 \times 10^{2}$ conidia $/ \mathrm{cm}^{2}$ that was comparable to the $99.1 \pm 7.4 \times 10^{2}$ conidia/ $\mathrm{cm}^{2}$ produced by the WT (Fig. $4 \mathrm{C}$ ). 
Given a significant increase in the FAE1 transcript levels also at the pre-invasive stage (12

405

406

407

408

409

410

411

412

413

414

415

416

417 hpi), we studied appressorial development in the fael $\Delta$ mutant. Appressorial assay carried out on an artificial hydrophobic surface showed that the morphology of the fael $\Delta$ appressoria was comparable to that of the WT appressoria observed at 24 hpi (Fig. 4D). We further quantified the appressorial development and found that the \% appressoria formed was similar in both the WT $(83.0 \pm 1.5 \%)$ and fael $\Delta$ mutant $(81.7 \pm 0.82 \%)$ (Fig. 4E). These results indicate that Fae1 does not play an important role in vegetative growth, asexual development and host-independent early pathogenic development in M. oryzae.

Next, we tested the pathogenicity of the fae $1 \Delta$ mutant on the host, where rice or barley whole plants were spray-inoculated with conidia harvested from the WT, fael $\Delta$ or fael $\triangle / F A E 1$ strain and incubated under humid conditions for 5-6 days. Interestingly, the fae $1 \Delta$-inoculated plants did not show typical blast disease lesions indicating that the mutant was significantly reduced in pathogenesis when compared with the WT or fae $1 \triangle / F A E 1$ strain (Fig. 5A and Supplementary Fig. S4A).

To further investigate the impaired pathogenesis in the fael $\Delta$ strain, we studied the invasive growth, if any, of the mutant by microscopically observing the rice sheath inoculated with the WT or mutant. We found that most fael $\Delta$ appressoria were unable to form visible primary invasive hyphae in rice sheath tissue (Fig. 5B). While $77.33 \pm 5.41 \%$ and $56.88 \pm 2.29 \%$ of the WT and fael $\triangle / F A E 1$ appressoria, respectively, formed clearly visible invasive hyphae, only $3.28 \pm 0.25 \%$ fae $1 \Delta$ appressoria were able to invade and form primary invasive hyphae $(P<0.001 ;$ Fig. 5C). Further, importantly, the primary invasive hyphae formed by the fael $\Delta$ mutant were restricted in the first invaded host cell and failed to elaborate to the neighboring cells (Fig. 5B). In order to rule out the possibility of delayed colonisation by the fael $\Delta$ mutant, we checked the invasive growth at 96 hpi. Indeed, even after prolonged incubation, 
the fae $1 \Delta$ mutant failed to colonize the plant tissue, as opposed to profuse invasive hyphal growth of the WT by then (Supplementary Fig. S3A). Similar phenotypes were also observed for the fae $1 \Delta$ mutant on other host plants such as barley and wheat (Supplementary Fig. S4B and S4C). Given that the Fae activity on the plant cell wall would release ferulic acid and polysaccharide molecules, we wondered whether exogenous supply of these compounds would support the fael $\Delta$ mutant in efficient host tissue colonisation. We performed an in vitro host-invasion assay on rice sheath inoculated with fae $1 \Delta$ mutant with $2 \%$ glucose or 100 $\mathrm{mM}$ ferulic acid, added at $22 \mathrm{hpi}$ (just before the onset of host penetration), and observed at $\sim 48$ hpi (Fig. 5B and Supplementary Fig. S3B). Indeed, while exogenously supplied glucose significantly rescued the fae $1 \Delta$ strain, addition of ferulic acid moderately $(42.9 \pm 4.6 \% ; P<$ 0.001) restored the host invasion and colonisation ability in the mutant (Fig. 5C). This observation was further substantiated by the whole-plant infection assay wherein the rice plants inoculated with the fael $\Delta$ mutant showed development of disease lesions upon exogenous addition of glucose or ferulic acid (Fig. 5B). To find out whether ferulic acid served as an antioxidant, we inoculated both leaf and sheath tissues with the fael $\Delta$ mutant in the presence of $20 \mathrm{mM} \mathrm{GSH}$, which is a known antioxidant. However, unlike ferulic acid, exogenous addition of GSH could not rescue the fael $\Delta$ mutant (Fig. 6A and 6B). Hence, we wondered whether M. oryzae could utilize ferulic acid as an energy source, since often small phenolic compounds such as ferulic acid can be used by fungi as an alternative or weak energy source (Black and Dix, 1976). Indeed, we found that M. oryzae WT grew on the basal medium supplemented with ferulic acid as the sole carbon source (Fig. 6C). This also possibly explains how exogenous addition of ferulic acid could rescue the fae $1 \Delta$ strain, most likely by providing energy to support invasive growth rather than acting as an antioxidant.

Altogether, our results show that the Fae1-based likely plant cell wall degradation is required specifically for successful host-invasion and colonisation during pathogenesis in M. oryzae. 


\section{Discussion}

Plant cell wall degrading enzymes (CWDEs) play a pivotal role in virulence of catalyze substrate hydrolysis following the mechanism of serine proteases having a conserved motif GXSXG and a conserved Ser-His-Asp/Glu catalytic triad (Dilokpimol et al., 2016). Our in-silico analysis of putative Fae in M. oryzae showed the presence of conserved GXSXG motif and were found to belong to $\alpha / \beta$-hydrolase-fold superfamily.

Often, CWDEs are found as an expanded gene family in phytopathogenic fungi and are difficult to specifically characterize mainly due to tight transcriptional regulation and functional redundancy of the members of the gene family i.e., loss of function of one gene is often compensated by the other genes in the family. Our HMM-based domain analysis across different fungal species showed a significant genetic diversity in the Fae sequences therein. Importantly, absence of any putative Fae in budding and fission yeasts suggests that the enzyme from other, especially phytopathogenic, fungal species could be associated mainly with degradation of plant cell walls. Similarly, presence of large number of Fae sequences in non-pathogenic filamentous fungi such as Aspergillus spp. and N. crassa is intriguing and studies on FAEs across different fungal genera could possibly shed some light on any evolutionary aspect of it. Indeed, our phylogenetic analysis revealed that the $M$. oryzae Fae1 (MGG_08737) has evolutionarily diverged more as compared to its paralogs and orthologs across different fungi. In $M$. oryzae, previous studies were aimed at understanding the role of endo-xylanases (Nguyen et al., 2011) and cellulases (Vu et al., 2012) in fungal pathogenesis, 
by simultaneous silencing of multiple genes. Similarly, Cutinase2 (one out of the three putative Cutinases), which was significantly induced during host penetration stage, plays a role in full virulence in M. oryzae (Skamnioti and Gurr, 2007). In the present study, we investigated the role of one of the feruloyl esterases (Fae1) in pathogenicity of M. oryzae.

We found that the extracellular feruloyl esterase activity in the blast fungus was significantly increased in the presence of rice leaf extract. Possibly, FAEs were induced by the complex mixture of host factor(s) including the individual plant cell wall components. Thus, we studied the expression pattern of all the $F A E$ genes under host- or pathogenicity-mimic conditions. We found that $F A E$ genes expressed differentially, with majority of them accumulating $>1.5$ fold higher, in response to individual plant cell wall components. While our observation is consistent with a previously reported similar upregulation of FAEs in Aspergillus niger (de Vries et al., 2002) and Fusarium graminearum (Balcerzak et al., 2012), it remains to be tested whether a combination of more than one host cell wall component would cause further upregulation in $F A E$ expression Intriguingly, majority of the FAEs were significantly upregulated in the presence of $\mathrm{N}$-acetylglucosamine (NAG). A secreted $M$. oryzae chitinase (MoChia1), that binds to chitin to suppress plant immune response during infection (Yang et al., 2019), could likely digest free chitin to monomeric NAG, which in turn could be sensed by the blast fungus to express Fae. However, this hypothesis needs to be tested further.

A recent study on transcriptome profiling showed that MGG_08737 significantly upregulates at 18 hpi (161-fold), 27 hpi (82-fold), 36 hpi (130-fold), 45 hpi (147-fold) and 72 hpi (228fold) during infection cycle of M. oryzae (Jeon et al., 2020). We found a similar pattern, where FAE1 was specifically upregulated during both pre-invasive appressorial development 
(12 and $24 \mathrm{hpi}$ ) and post-penetration host colonisation stages (48 to $72 \mathrm{hpi}$ ) of the infection cycle. Accordingly, loss of Fae1 function specifically impaired the ability of M. oryzae to invade and colonize rice, barley and wheat tissue. Although our in silico analysis suggested that the Fae function could be attributed to specific hosts, the defect in the fael $\Delta$ mutant could not be correlated to any of the host species used in this study. However, it remains to be studied whether fael $\Delta$ has a distinct phenotype with any other cereal crops. Further, very few appressoria $(<5 \%)$ that were able to form invasive hyphae in the mutant were defective in spreading to the adjacent host cells and were rather restricted to the first cell invaded, even after prolonged incubation (96 hpi). Our observations are in line with the hypothesis that Fae, and CWDEs in general, likely play an important role in cell-to-cell spread of the fungus within the host tissue, and subsequent necrotrophic growth phase (Zheng et al., 2009). We were intrigued by our observation that the deletion of just one $F A E$ led to a significant defect in host invasion and that the presence of none other putative FAE could compensate for the loss of Fae1 function. Interestingly, our in silico analysis suggests that Fae1, among all the $M$. oryzae FAEs, is highly diverged (Fig. 1B) and that there is no significant identity between any two Fae proteins in M. oryzae (Supplementary Fig. S5). This might possibly explain why the loss-of-function of Fae1 alone led to a significant phenotype in M. oryzae.

During appressorial development, the blast fungus derives energy by utilizing stored lipids via $\beta$-oxidation in the mitochondria and peroxisomes, generating acetyl-CoA, which is further distributed into the glyoxylate cycle and gluconeogenesis (Wang et al., 2007; Patkar et al., 2012). It is hypothesized that this metabolic process might be required to support the initial appressorial development and maturation, which ensures host penetration by the blast fungus, and the subsequent energy requirement during host tissue colonisation could be fulfilled by the host-derived nutrients (Fernandez and Wilson, 2014). It is possible that the plant cell wall 
carbohydrates released after CWDEs activity could act as an energy source for the fungus at the host-pathogen interface and facilitate its entry and/or elaboration into the host. Feruloyl esterases in Aspergillus niger act synergistically, with other CWDEs like cellulases, xylanases and pectinases, to degrade the complex plant cell wall carbohydrates (Faulds and Williamson, 1995). In M. oryzae, endo-xylanases (Nguyen et al., 2011) and cellulases (Vu et al., 2012) are shown to be important in host-penetration and virulence of the blast fungus. Considering all this, we wondered whether sugar released from CWDE-mediated plant cell wall digestion could act as an energy source for the fungus. Indeed, exogenous supply of glucose rescued the fae $1 \Delta$ strain, indicating that the mutant was deprived of energy source at the time of host invasion. Interestingly, exogenous supply of ferulic acid, which is a product of Fae enzyme action, could also moderately support the invasive growth of the fael $\Delta$ mutant. This is in accordance with a previous hypothesis that ferulic acid could possibly act as a weak or an alternative carbon source (Black and Dix, 1976; Fig. 6C). Here, we propose that, like in A. niger, the Fae in M. oryzae acts, in a concerted manner, on the esterified ferulic acid bridges in the plant cell wall to allow endo-xylanases and cellulases to work on the carbohydrates therein, releasing constituent sugar molecules and ferulic acid, which could act as the energy source during host invasion (Fig. 7).

Feruloyl esterases have a wide range of applications in biofuel industry, food, cosmetic and pharmaceutical industry and also paper and pulp industry, all of which involve plant biomass

547 degradation (Dilokpimol et al., 2016). It is often used in conjunction with other plant cell wall deconstructing enzymes. Most of the applied aspects of feruloyl esterases have been studied in Aspergillus spp. Although M. oryzae is a phytopathogenic fungus, one could explore the potential use of recombinantly expressed Fae1 in industrial applications, both in terms of enzyme activity and range of substrate specificity. Similarly, ferulic acid, the 
552

553

554

555

556

557

558

559

560

561

562

563

564

565

566

567

568

569

570

571

572

573

574

575

product of feruloyl esterase enzyme action, has a large application in food and pharmaceutical industry (Dilokpimol et al., 2016). Thus, use of an efficient feruloyl esterase (M. oryzae Fae1) for production of ferulic acid could also be explored. Moreover, it has been reported that Fae can also act on synthetic esterified substrates such as methyl ferulate, methyl sinapate, methyl $p$-coumarate and methyl caffeate (Crepin et al., 2004). Therefore, it would be worth exploring whether or not Fae1, or feruloyl esterases in general, can also act on rutin complexed with glucose, thereby releasing quercetin, a plant flavanol with medicinal properties. This might also implicate another potential commercial application of Fae.

Further, given the involvement of Fae1 specifically in pathogenicity of $M$. oryzae, it could be considered as a potential target for developing an antifungal strategy.

Altogether, we show that the M. oryzae feruloyl esterase Fae1 plays a key role in pathogenesis, wherein the enzyme activity likely makes the alternative energy source available and supports the fungal growth during host invasion and colonisation.

\section{Acknowledgements}

We acknowledge late Prof. Bharat B. Chattoo for his guidance and providing sophisticated laboratory facility at the Bharat Chattoo Genome Research Centre (BCGRC). We also fondly remember late Dr. Johannes Manjrekar for the useful scientific discussions during the course of this work. We thank Naweed Naqvi (Temasek Lifesciences Laboratory, Singapore) for providing backbone vector pFGL1010. AT was supported by UGC-BSR-RFSMS (F.7128/2007-BSR, dtd-02.09.2014), University Grants Commission, Government of India; and KM acknowledges the intramural University Research Scholarship from The M. S. 
576 University of Baroda. This work was supported by the Ramalingaswami Fellowship, DBT,

577 GoI (BT/RLF/Re-entry/32/2014) awarded to RP. We thank the BCGRC group at MSU for 578 useful discussions.

579

580 Author contributions

581 Conceptualization: AT and RP; Methodology: AT and RP; Validation: AT and KM; Formal 582 analysis: AT; Investigation: AT and KM; Resources: RP; Writing - original draft: AT and

583 KM; Writing - review and editing: RP; Supervision: RP; Project administration: RP; Funding 584 acquisition: RP.

585

586 Declarations:

587 Competing interests - Authors declare that there are no competing interests involved in this 588 work.

589 Ethics approval - Not applicable.

590 Consent to participate - Not applicable.

591 Consent for publication - Not applicable.

592

Reference:

1. Balcerzak, M., Harris, L. J., Subramaniam, R., Ouellet, T., 2012. The feruloyl esterase gene family of Fusarium graminearum is differentially regulated by aromatic compounds and hosts. Fungal Biol. 116: 478-488. 
2. Biswas, S., Dijck, P.V., Datta, A., 2007. Environmental sensing and signal transduction pathways regulating morphopathogenic Determinants of Candida albicans. Microbiol. Mol. Biol. Rev. 71: 348-376.

3. Black, R. L. B., Dix, N. J., 1976. Utilization of ferulic acid by microfungi from litter and soil. Transact. British Mycol. Soc. 66: 313-317.

4. Bunzel, M., Ralph, J., Marita, J. M., Hatfield, R. D., Steinhart, H., 2001. Diferulates as structural components in soluble and insoluble cereal dietary fibre. J. Sci. Fd. Agric. 81: 653-660.

5. Capella-Gutiérrez, S., Silla-Martínez, J. M., Gabaldón, T., 2009. trimAl: A tool for automated alignment trimming in large-scale phylogenetic analyses. Bioinformatics 25: $1972-1973$.

6. Cosgrove, D. J., 2001. Wall structure and wall loosening. A look backwards and forwards. Plant Physiol. 125: 131-134.

7. Crepin, V. F., Faulds, C. B., Connerton, I. F., 2004. Functional recognition of new classes of feruloyl esterases. Appl. Microbiol. Biotechnol. 63: 647-652.

8. De Vries, R. P., Michelsen, B., Poulsen, C. H., Kroon, P. A., van den Heuvel, R. H. H., et al., 1997. The faeA genes from Aspergillus niger and Aspergillus tubingensis encode ferulic acid esterases involved in degradation of complex cell wall polysaccharides. Appl. Environ. Microbiol. 63: 4638-4644.

9. De Vries, R. P., Vankuyk, P. A., Kester, H. C. M., Visser, J., 2002. The Aspergillus niger faeB gene encodes a second feruloyl esterase involved in pectin and xylan degradation and is specifically induced in the presence of aromatic compounds. Biochem. J. 363: 377-386.

10. Dean, R. A., Talbot, N. J., Farman, M. L., Mitchell, T. K., Orbach, M. J., et al., 2005. The genome sequence of the rice blast fungus Magnaporthe grisea. Nature 434: 980 986.

11. Dellaporta, S. L., Wood, J., Hicks, J. B., 1983. A plant DNA minipreparation: Version II. Plant Mol. Biol. Rep. 1: 19-21.

12. Dilokpimol, A., Makela, M. R., Anguilar-Pontes, M. V., Benoit-Gelber, I., Hidden, K. S., et al., 2016. Diversity of fungal feruloyl esterases: updated phylogenetic classification, properties, and industrial applications. Biotechnol. Biofuels 9: 231.

13. Ebbole, D. J., 2007. Magnaporthe as a model for understanding host-pathogen interactions. Annu. Rev. Phytopathol. 45: 437-456.

14. Faulds, C. B., Williamson, G., 1994. Purification and characterization of a ferulic acid esterase (FAEIII) from Aspergillus niger: specificity for the phenolic moiety and binding to micro-crystalline cellulose. Microbiology 140: 779-787.

15. Faulds, C. B., Williamson, G., 1995. Release of ferulic acid from wheat bran by a ferulic acid esterase (FAE-III) from Aspergillus niger. Appl. Microbiol. Biotechnol. 43: $1082-1087$. 
16. Fernandez, J., Wilson, R. A., 2014. Cells in cells: morphogenetic and metabolic strategies conditioning rice infection by the blast fungus Magnaporthe oryzae. Protoplasma 251: 37-47.

17. Gietz, R. D., Woods, R. A., 2002. Transformation of yeast by lithium acetate/singlestranded carrier DNA/polyethylene glycol method. Methods Enzymol. 350: 87-96.

18. Hancock, J. M., Bishop, M. J., 2004. HMMer. In Dictionary of Bioinformatics and Computational Biology (Vol. 1-June). John Wiley \& Sons, Ltd.

19. Harris, P. J., Hartley, R. D., 1977. Detection of bound ferulic acid in cell walls of the Gramineae by ultraviolet fluorescence microscopy. Nature 259: 508-510.

20. Hermoso, J. A., Aparicio, J. S., Molina, R., Juge, N., Gonzalez, R., et al., 2004. The crystal structure of Feruloyl esterase A from Aspergillus niger suggests evolutive functional convergence in feruloyl esterase family. J. Mol. Biol. 338: 495-506.

21. Howard, R. J., Ferrari, M. A., Roach, D. H., Money, N. P., 1991. Penetration of hard substrates by a fungus employing enormous turgor pressure. Proc. Natl. Acad. Sci. U.S.A. 88: $11281-11284$.

22. Jeon, J., Lee, G. W., Kim, K. T., Park, S. Y., Kim, S., et al., 2020. Transcriptome profiling of the rice blast fungus Magnaporthe oryzae and its host Oryza sativa during infection. Mol. Plant Microbe Interact. 33: 141-144.

23. Kachroo, P., Lee, K. H., Schwerdel, C., Bailey, J. E., Chattoo, B. B., 1997. Analysis of host-induced response in the rice blast fungus Magnaporthe grisea using twodimensional polyacrylamide gel electrophoresis. Electrophoresis. 18: 163-169.

24. Kachroo, P., Leong, S. A., Chattoo, B. B., 1994. Pot2, an inverted repeat transposon from the rice blast fungus Magnaporthe grisea. Mol. Gen. Genet. 245: 339-348.

25. Kubicek, C. P., Starr, T. L., Glass, N. L., 2014. Plant cell wall-degrading enzymes and their secretion in plant-pathogenic fungi. Annu. Rev. Phytopathol. 52: 427-451.

26. Kumar, S., Stecher, G., Tamura, K., 2016. MEGA7: Molecular Evolutionary Genetics Analysis Version 7.0 for Bigger Datasets. Mol. Biol. Evol. 33: 1870-1874.

27. Lee, S., Kelley, B. S., Damasceno, C. M. B., St. John, B., Kim, B., et al., 2006. A functional screen to characterize the secretomes of eukaryotic pathogens and their hosts in planta. Mol. Plant Microbe Interact. 19: 1368-1377.

28. Letunic, I., Bork, P., 2019. Interactive Tree Of Life (iTOL) v4: Recent updates and new developments. Nucleic Acids Res. 47(W1): W256-W259.

29. Livak, K. J., Schmittgen, T. D., 2001. Analysis of relative gene expression data using real-time quantitative PCR and the $2^{-\Delta \Delta C \mathrm{~T}}$ method. Methods 25: 402-408.

30. Marchler-Bauer, A., Derbyshire, M. K., Gonzales, N. R., Lu, S., Chitsaz, F., et al., 2015. CDD: NCBI's conserved domain database. Nucleic Acids Res. 43(Database issue): D222-D226.

31. Mastihuba, V., Kremnicky, L., Mastihubova, M., Willett, J. L., Cote, G. L., 2002. A spectrophotometric assay for feruloyl esterases. Anal. Biochem. 309: 96-101. 
32. Nguyen, L.-T., Schmidt, H. A., von Haeseler, A., Minh, B. Q., 2015. IQ-TREE: A Fast and Effective Stochastic Algorithm for Estimating Maximum-Likelihood Phylogenies. Mol. Biol. Evol. 32: 268-274.

33. Nguyen, Q. B., Itoh, K., Vu, V. B., Tosa, Y., Nakayashiki, H., 2011. Simultaneous silencing of endo- $\beta-1,4$ xylanase genes reveals their roles in the virulence of Magnaporthe oryzae. Mol. Microbiol. 81: 1008-1019.

34. Patkar, R. N., Benke, P. I., Qu, Z., Chen, Y., Yang, F., et al., 2015. A fungal monooxygenase-derived jasmonate attenuates host innate immunity. Nat. Chem. Biol. 11: 733-740.

35. Patkar, R. N., Ramos-Pamplona, M., Gupta, A. P., Fan, Y., Naqvi, N. I., 2012. Mitochondrial $\beta$-oxidation regulates organellar integrity and is necessary for conidial germination and invasive growth in Magnaporthe oryzae. Mol. Microbiol. 86: 13451363.

36. Patkar, R. N., Suresh, A., Naqvi, N. I., 2010. MoTea4-mediated polarized growth is essential for proper asexual development and pathogenesis in Magnaporthe oryzae. Eukaryot. Cell 9: 1029-1038.

37. Petersen, T. N., Brunak, S., Heijne, G., Nielsen, H., 2011. SignalP 4.0: discriminating signal peptides from transmembrane regions. Nat. Methods 8: 785-786.

38. Prakash, C., Manjrekar, J., Chattoo, B. B., 2016. Skp1, a component of E3 ubiquitin ligase, is necessary for growth, sporulation, development and pathogenicity in rice blast fungus (Magnaporthe oryzae). Mol. Plant Pathol. 17: 903-919.

39. Sievers, F., Higgins, D. G., 2018. Clustal Omega for making accurate alignments of many protein sequences. Protein Sci. 27: 135-145.

40. Sievers, F., Wilm, A., Dineen, D., Gibson, T.J., Karplus, K., et al., 2011. Fast, scalable generation of high-quality protein multiple sequence alignments using Clustal Omega. Mol. Syst. Biol. 7: 539.

41. Skamnioti, P., Gurr, S. J., 2007. Magnaporthe grisea Cutinase2 mediates appressorium differentiation and host penetration and is required for full virulence. Plant Cell 19: 2674-2689.

42. Skamnioti, P., Gurr, S. J., 2008. Against the grain: safeguarding rice from rice blast disease. Trends Biotechnol. 27: 141-150.

43. Soundararajan, S., Jedd, G., Li, X., Ramos-Pamploña, M., Chua, N. H., et al., 2004. Woronin body function in Magnaporthe grisea is essential for efficient pathogenesis and for survival during nitrogen starvation stress. The Plant cell 16: 1564-1574.

44. Valent, B., Khang, C. H., 2010. Recent advances in rice blast effector research. Curr Opin Plant Biol. 13: 434-441.

45. Vu, B. V., Itoh, K., Nguyen, Q. B., Tosa, Y., Nakayashiki, H., 2012. Cellulases belonging to glycoside hydrolase families 6 and 7 contribute to the virulence of Magnaporthe oryzae. Mol. Plant Microbe Interact. 25: 1135-1141. 
46. Wang, Z. Y., Soanes, D. M., Kershaw, M. J., Talbot, N. J., 2007. Functional analysis of lipid metabolism in Magnaporthe grisea reveals a requirement for peroxisomal fatty acid beta-oxidation during appressorium-mediated plant infection. Mol. Plant Microbe Interact. 20: 475-491.

47. Xu, M., Gao, X., Chen, J., Yin, Z., Feng, H., Huang, L., 2017. The feruloyl esterase genes are required for full pathogenicity of the apple tree canker pathogen Valsa mali. Mol. Plant Pathol. 19: 1353-1363.

48. Yang, C., Yu, Y., Huang, J., Meng, F., Pang, J., et al., 2019. Binding of the Magnaporthe oryzae chitinase MoChial by a rice tetratricopeptide repeat protein allows free chitin to trigger immune responses. Plant Cell 31: 172-188.

49. Yang, F., Naqvi, N., 2014. Sulfonylurea resistance reconstitution as a novel strategy for ILV2-specific integration in Magnaporthe oryzae. Fungal Genet Biol. 68: 71-6.

50. Yu, J. H., Hamari, Z., Han, K. H., Seo, J. A., Reyes-Domínguez Y, et al., 2004. Double-joint PCR: a PCR-based molecular tool for gene manipulations in filamentous fungi. Fungal Genet. Biol. 41: 973-981.

51. Zheng, X., Zhou, J., Lin, C., Lin, X., Lan, L., et al., 2009. Secretion property and gene expression pattern of a putative feruloyl esterase in Magnaporthe grisea. In: Xiaofan, W., Valent, B. (eds), Advances in genetics, genomics and control of rice blast disease, $1^{\text {st }}$ edn. Springer, Netherlands, pp. 41-50. 
Figure 1. Identification and phylogenetic analysis of putative fungal Fae. (A) Multiple sequence alignment of putative Fae sequences from $M$. oryzae, A. oryzae and $N$. crassa. The conserved GXSXG motif is marked with a red box. (B) Phylogenetic analysis of Fae sequences from 21 fungal species representing seven classes. The phylogenetic tree is based on maximum likelihood methods, with assessment of branch support by 1000 replicates of ultrafast bootstrap. Tree branches are color-coded according to the specific fungal species. The colors in the outer and inner circular strips represent taxonomy, for each branch, with respect to fungal class and species, respectively. Bootstrap values are indicated as grey circles, sized according to the values. Grey highlighted background represents the sub-clades belonging to the Magnaporthales order. Stars denote Fae proteins from M. oryzae; filled-star marks the MGG_08737 gene used in the present study. Stars in clockwise direction - putative Fae encoded by MGG_09677, MGG_09732, MGG_03502, MGG_02261, MGG_05592, MGG_05366, MGG_09404, MGG_03771, and MGG_08737, respectively. (C) Phylogenetic tree depicting combined analysis of Fae sequences in host-specific M. oryzae isolates from rice (Oryza; pink), wheat (Triticum; blue), foxtail millet (Setaria; purple) and finger millet (Eleusine; green).

Figure 2. M. oryzae feruloyl esterases are secretory in nature and induced by host leaf extract. (A) A schematic representation of the Yeast Secretion Trap strategy used to study presence of a signal peptide in Fae. (B) The Yeast Secretion Trap based assay depicting growth of the transformants expressing M. oryzae FAE(MGG_05529 or MGG_07294) when compared to the no-growth, on SD + Sucrose, of the recipient strain (DBY $\alpha 2445)$ with or without the backbone vector (pYST). (C) A bar chart showing Fae enzyme activity in the 
intracellular versus extracellular (secretory) fraction of the M. oryzae culture grown in the presence or absence of rice leaf extract. The data is represented as mean \pm s.d.m. from three independent experiments. ${ }^{* * *}, P<0.001$.

\section{Figure 3. Differential expression of feruloyl esterase genes during pathogenesis in $M$.}

oryzae. (A) A bar chart showing relative transcript levels of nine FAEs in M. oryzae vegetative culture grown under different conditions. The cultures were grown in minimal medium with either $0.03 \%$ cutin monomers $(1,16$ - hexadecanediol), $0.03 \%$ ferulic acid, nitrogen-starvation, $1 \%$ Pectin, $1 \% \mathrm{~N}$-acetylglucosamine (NAG) or 1\% Xylan, for $48 \mathrm{~h}$ before harvesting the biomass. The $F A E$ transcript levels were estimated relative to those in the vegetative culture grown in minimal medium containing $1 \%$ glucose as a control condition. The horizontal line corresponding to the fold change 1 represents normalized transcript levels for the control condition. (B) A bar chart depicting relative transcript levels of nine FAEs during different stages of pathogenic development. Samples were harvested at the time points mentioned and the transcript levels were compared to those from vegetative mycelia as control condition. $\beta$-tubulin was used as an endogenous control in both (A) and (B). Data represents mean values \pm s.d.m. from three independent biological replicates with technical triplicates each time.

\section{Figure 4. Fae1 function is not required for vegetative and host-independent early} pathogenic development in M. oryzae. Vegetative growth of the WT or fael $\Delta$ on prune agar plates at 10 dpi (A) with measurements of diameter of colonies of both the strains shown in (B). Data represents mean \pm s.d.m. from the experiments repeated thrice. (C) A bar graph depicting total conidiation assessed by counting the number of conidia harvested at $10 \mathrm{dpi}$ 
from the WT or fael $\Delta$ culture grown on PA plates. Data represents mean \pm s.d.m. from three

782

783

784

785

786

787 independent experiments. ns, not significant. (D) Micrographs showing appressorial development, on an inductive glass surface, in the WT or fael $\Delta$, at $24 \mathrm{hpi}$. Scale bar, $10 \mu \mathrm{m}$. (E) A quantitative analysis of appressorial development in the WT or fae $1 \Delta$ at 24 hpi. Data represents mean \pm s.d.m. from three independent experiments, with at least 100 appressoria each observed for quantification. ns, not significant.

\section{Figure 5. Fae1 function is crucial for host invasion and colonisation during blast}

disease. (A) Whole-plant infection assay depicting blast disease outcome from the rice plants spray-inoculated with either the WT, fae $1 \Delta$ or fae $1 \triangle / F A E 1$ conidia or fae $1 \Delta$ supplemented at 22 hpi with $2 \%$ glucose or $100 \mathrm{mM}$ ferulic acid. The representative leaves were detached and photographed after 6 dpi. (B) Micrographs showing host invasion ( 48 hpi) ability of either the WT, fael $\triangle$ mutant, fael $\triangle / F A E 1$ or fae $1 \Delta$ supplemented with $2 \%$ glucose or $100 \mathrm{mM}$ ferulic acid. Glucose or ferulic acid was added after 22 hpi. Images were taken at $\sim 48$ hpi. Asterisks mark appressoria, while arrowheads depict the invasive hyphae. Yellow asterisks mark the non-invading fael $\Delta$ appressoria and the arrow depicts the invasive hypha restricted to the first invaded host (rice sheath) cell. Scale bar, $10 \mu \mathrm{m}$. (C) A bar chart depicting percentage of appressoria invading the rice sheath inoculated with either the WT, fael $\Delta$, fae $1 \triangle / F A E 1$ or fae $1 \triangle$ supplemented with $2 \%$ glucose or $100 \mathrm{mM}$ ferulic acid. Data represents mean \pm s.d.m. from three independent experiments, with at least 100 appressoria each observed for quantification. ${ }^{* * *}, P<0.001$; ns, not significant.

Figure 6. Ferulic acid released by the Fae1 action is likely required as a nutrient source during host invasion and colonisation by M. oryzae. (A) Drop-inoculation assay showing 
infection ability (on detached rice leaves) of WT, fael $\Delta$ or fael $\Delta$ supplemented with $100 \mathrm{mM}$ ferulic acid (FA) or $20 \mathrm{mM}$ reduced glutathione (GSH). Images were taken at 6 dpi. (B) Leaf

807 sheath inoculation assay showing host invasion ability of the WT or fael $\Delta$ supplemented with $20 \mathrm{mM} \mathrm{GSH}$, which was added at $22 \mathrm{hpi}$. The results were observed at 48 hpi. White and yellow asterisks mark the invading and non-invading appressoria, respectively. Arrow depicts invasive hypha restricted to the first host cell invaded. Scale bar, $10 \mu \mathrm{m}$. (C)

811 Vegetative growth of the WT M. oryzae on basal medium (BM) with or without $0.01 \%$

812 ferulic acid. The images were taken at $10 \mathrm{dpi}$.

814 Figure 7. A proposed model of Fae1 function during pathogenesis in M. oryzae. Fae1,

815 likely secreted along with other CWDEs, hydrolyses the plant cell wall to release ferulic acid 816 and constituent carbohydrates during penetration of the first host cell and subsequent spread

817 to the neighbouring cells. Released ferulic acid, the product of Fae enzyme action, and/or

818 glucose, the breakdown product of cellulose, likely act as an energy source enabling

819 successful host-invasion and colonisation by the blast fungal pathogen. 


\section{Figures}

A

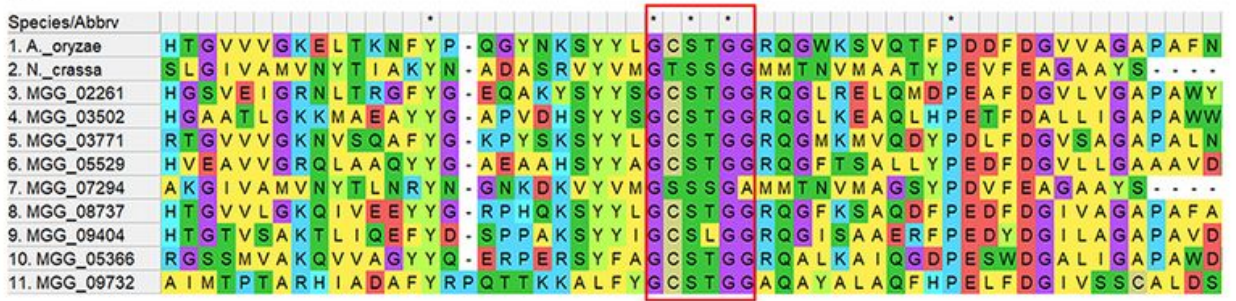

C Tree scale: 0.01

हू.

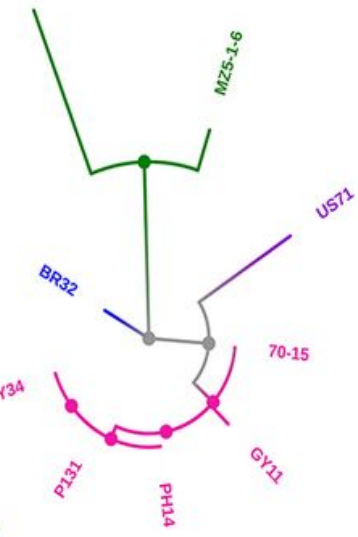

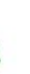

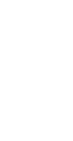

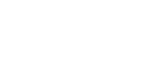

B

Species

Alternaria altemata

Aspergillus nidulan

Aspergillus niger

Aspergillus oryzae

Colletotrichum graminicola

Eremothecium cymbalariae

Fusarium graminearum

Fusarium oxysporum

Gaeumannomyces tritici

Magnaporthiopsis poae

Neurospora crassa

Magnaporthe grisea

Magnaporthe oryzae

Pyricularia pennisetigena

Saccharomyces cerevisiae

Schizosaccharomyces pombe

Sclerotinia sclerotiorum

Ustilago maydis

Valsa mali

Verticillium dahliae

Zymoseptoria tritici

\section{$\frac{\text { bootstrap }}{.20}$ \\ - 40 \\ - 60 \\ 80 \\ 100}

Class

Dothideomycetes

Eurotiomycetes

Leotiomycetes

Saccharomycetes

$\square$ Schizosaccharomycetes

Sordariomycetes

Ustilaginomycetes
Tree scale: 1
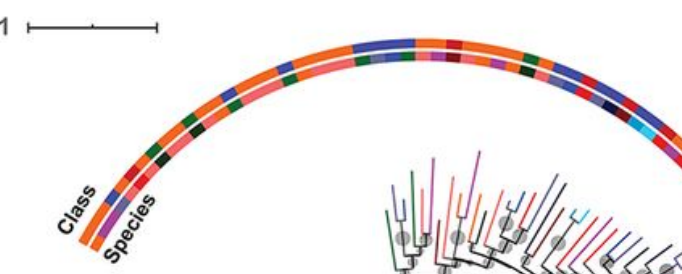

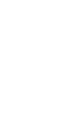
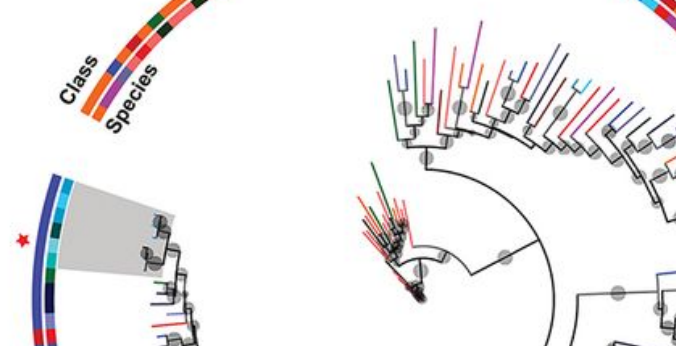

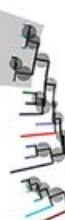

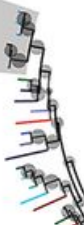

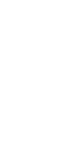


A

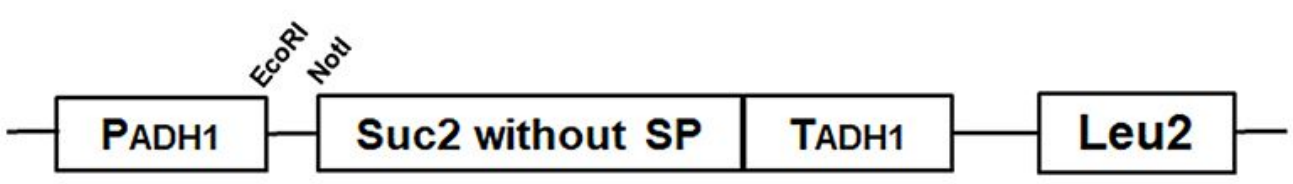

Clone the gene of interest upstream to Suc2

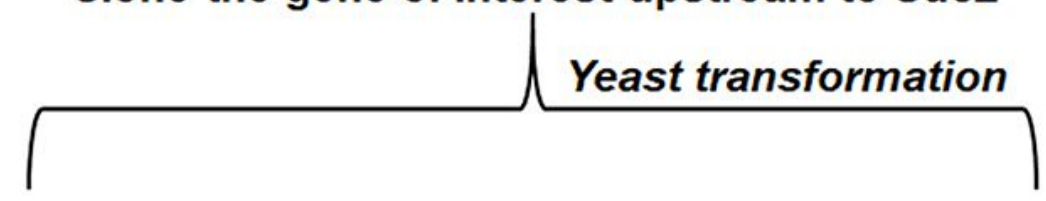

Gene without SP<smiles>[C]=C</smiles>

No growth on

sucrose medium

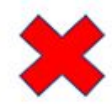

Gene with SP

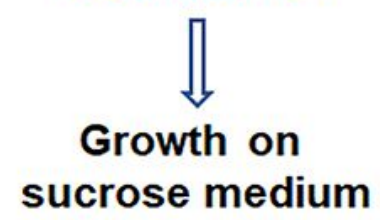

sucrose medium
B

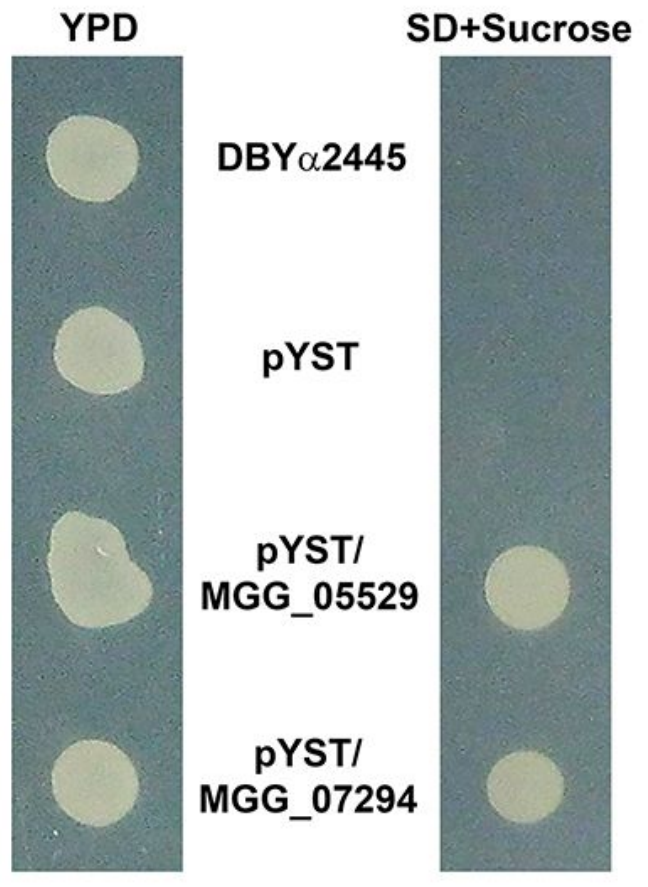

C

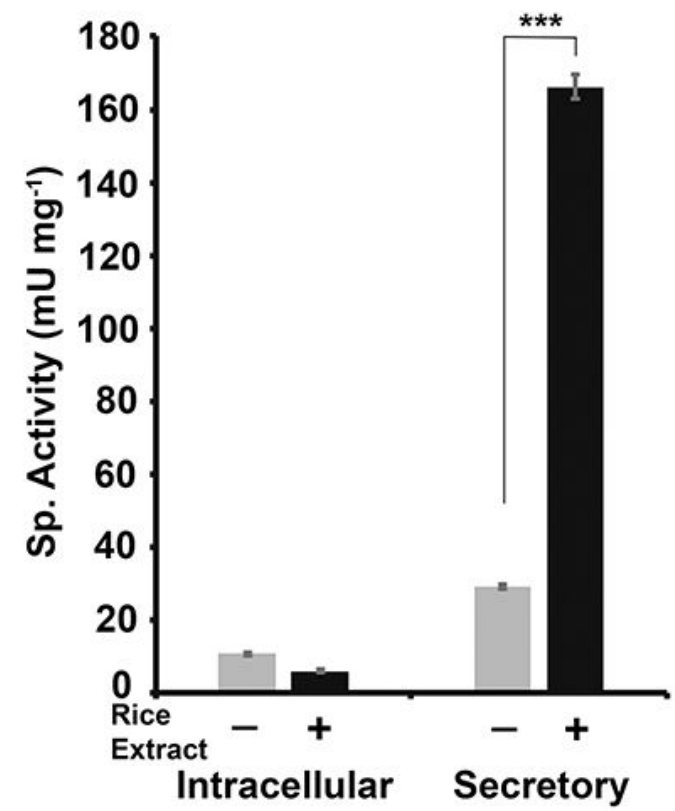

Figure 2

M. oryzae feruloyl esterases are secretory in nature and induced by host leaf extract. (A) A schematic representation of the Yeast Secretion Trap strategy used to study presence of a signal peptide in Fae. (B) The Yeast Secretion Trap based assay depicting growth of the transformants expressing M. oryzae FAE (MGG_05529 or MGG_07294) when compared to the no-growth, on SD + Sucrose, of the recipient strain (DBYa2445) with or without the backbone vector (pYST). (C) A bar chart showing Fae enzyme activity in 
the intracellular versus extracellular (secretory) fraction of the M. oryzae culture grown in the presence or absence of rice leaf extract. The data is represented as mean \pm s.d.m. from three independent experiments. ${ }^{* \star \star}, \mathrm{P}<0.001$.
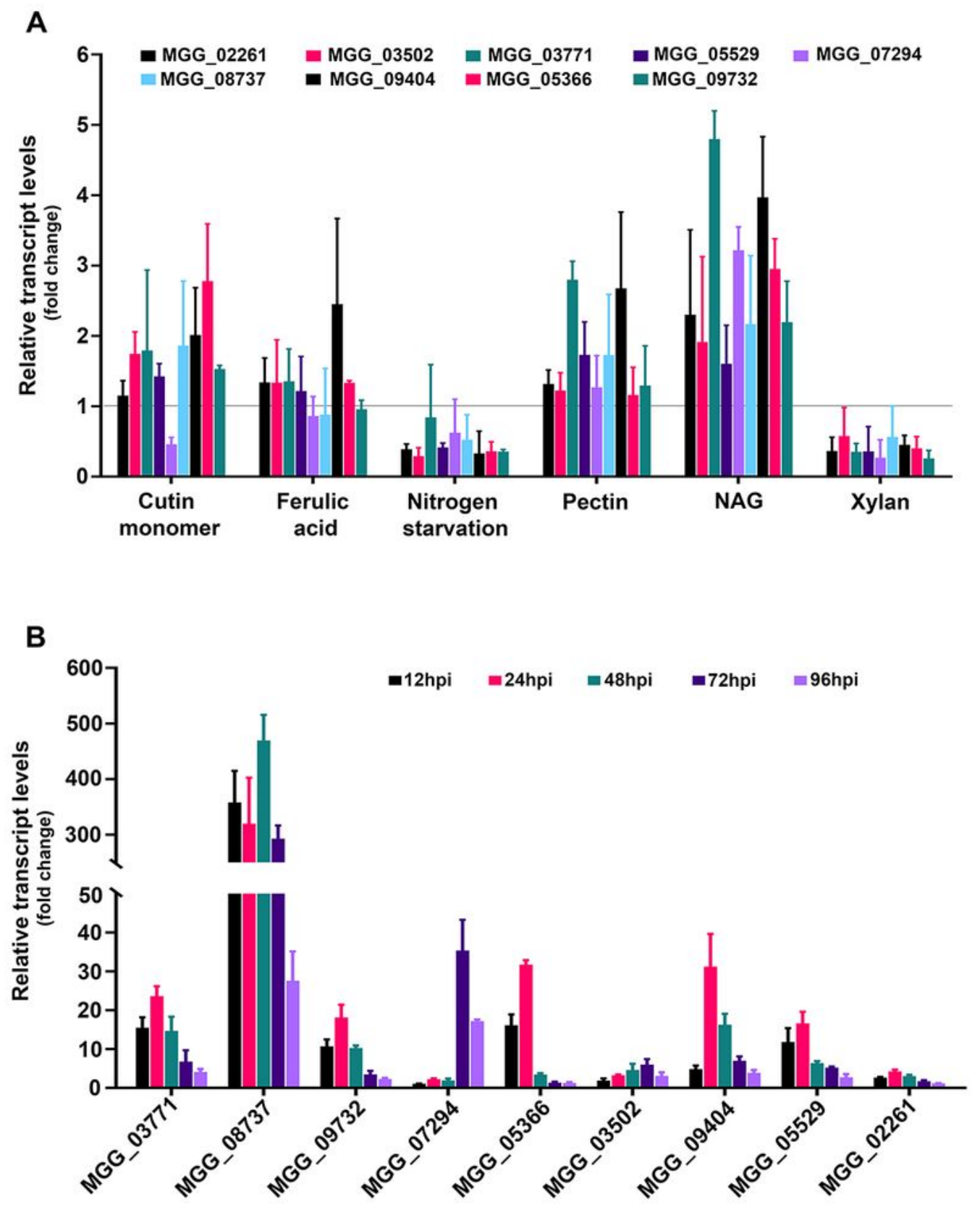

Figure 3

Differential expression of feruloyl esterase genes during pathogenesis in M. oryzae. (A) A bar chart showing relative transcript levels of nine FAEs in M. oryzae vegetative culture grown under different 
conditions. The cultures were grown in minimal medium with either $0.03 \%$ cutin monomers $(1,16$ hexadecanediol), $0.03 \%$ ferulic acid, nitrogen-starvation, $1 \%$ Pectin, $1 \%$ N-acetylglucosamine (NAG) or $1 \%$ Xylan, for $48 \mathrm{~h}$ before harvesting the biomass. The FAE transcript levels were estimated relative to those in the vegetative culture grown in minimal medium containing $1 \%$ glucose as a control condition. The horizontal line corresponding to the fold change 1 represents normalized transcript levels for the control condition. (B) A bar chart depicting relative transcript levels of nine FAEs during different stages of pathogenic development. Samples were harvested at the time points mentioned and the transcript levels were compared to those from vegetative mycelia as control condition. $\beta$-tubulin was used as an endogenous control in both $(A)$ and $(B)$. Data represents mean values \pm s.d.m. from three independent biological replicates with technical triplicates each time.

A

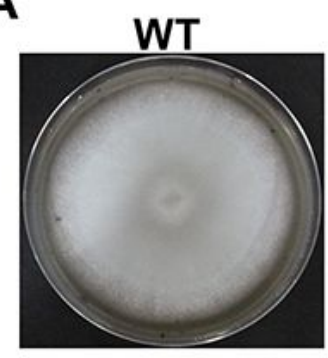

fae1 $1 \Delta$

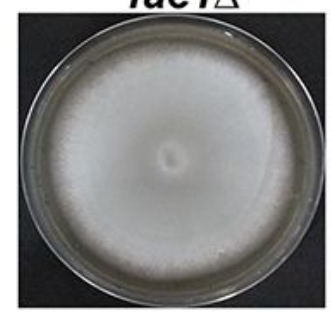

B

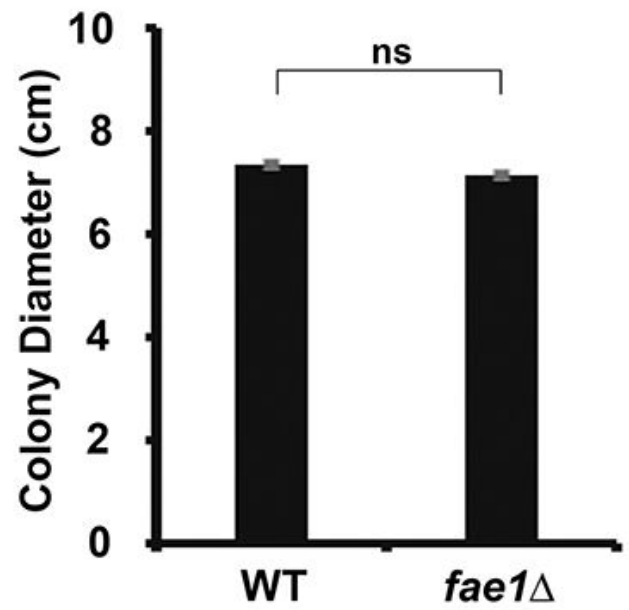

WT fae1s

C

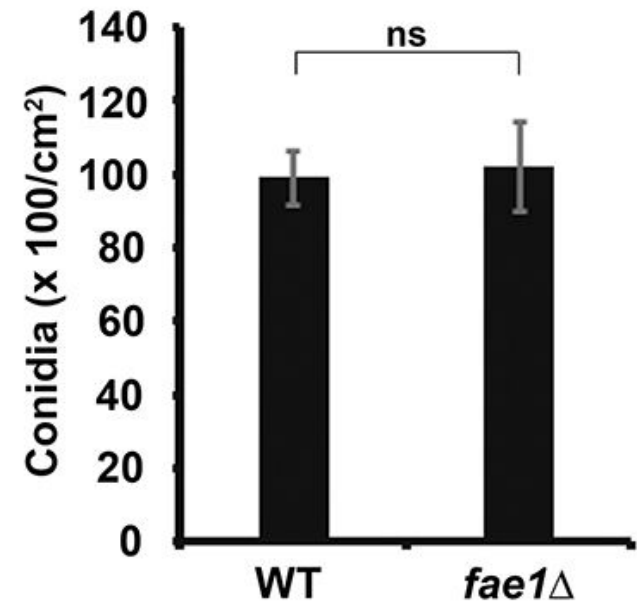

E

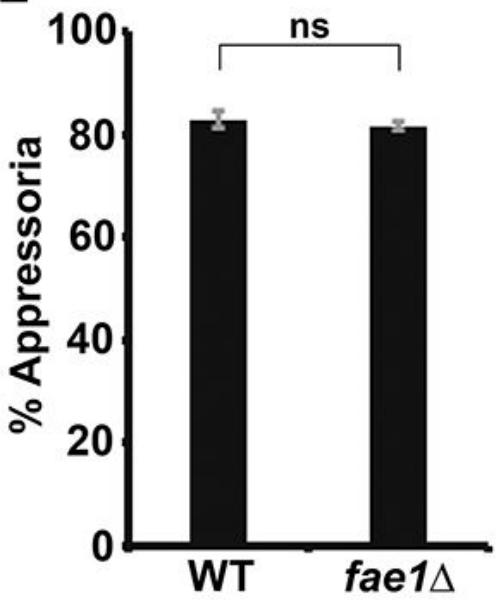

Figure 4

Fae1 function is not required for vegetative and host-independent early pathogenic development in $M$. oryzae. Vegetative growth of the WT or fae $1 \Delta$ on prune agar plates at $10 \mathrm{dpi}(\mathrm{A})$ with measurements of diameter of colonies of both the strains shown in (B). Data represents mean + s.d.m. from the 
experiments repeated thrice. (C) A bar graph depicting total conidiation assessed by counting the number of conidia harvested at $10 \mathrm{dpi}$ from the WT or fae $1 \Delta$ culture grown on PA plates. Data represents mean + s.d.m. from three independent experiments. ns, not significant. (D) Micrographs showing appressorial development, on an inductive glass surface, in the WT or fae1 $\Delta$, at $24 \mathrm{hpi}$. Scale bar, $10 \mu \mathrm{m}$. (E) A quantitative analysis of appressorial development in the WT or fae $1 \Delta$ at $24 \mathrm{hpi}$. Data represents mean \pm s.d.m. from three independent experiments, with at least 100 appressoria each observed for quantification. ns, not significant.

A

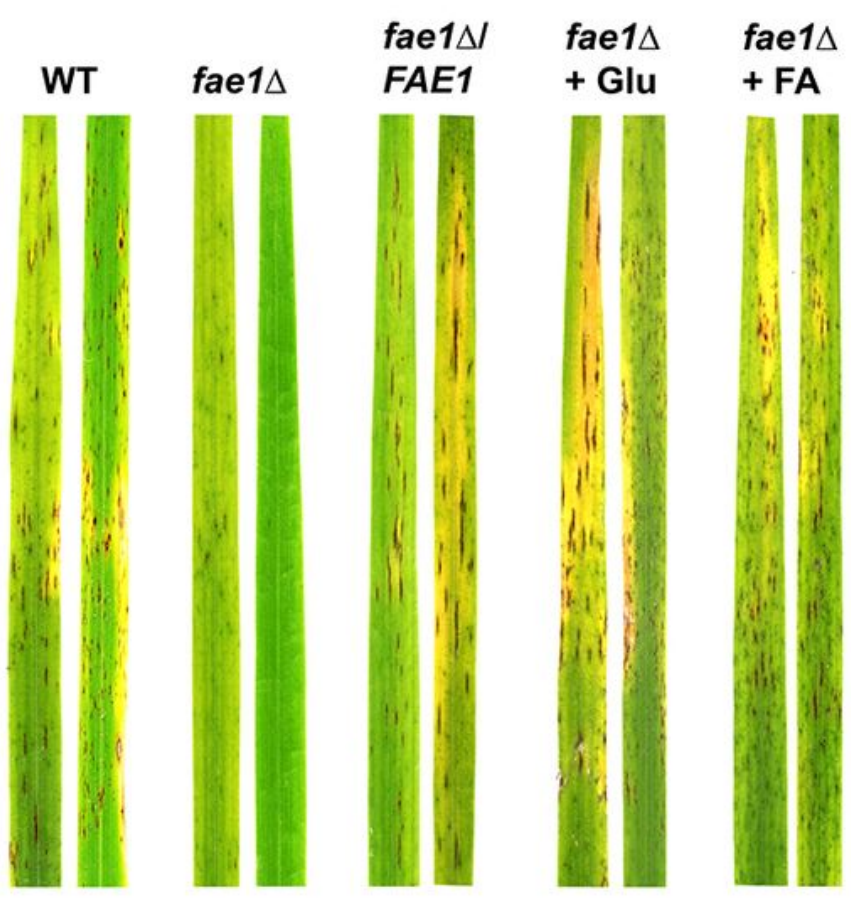

C

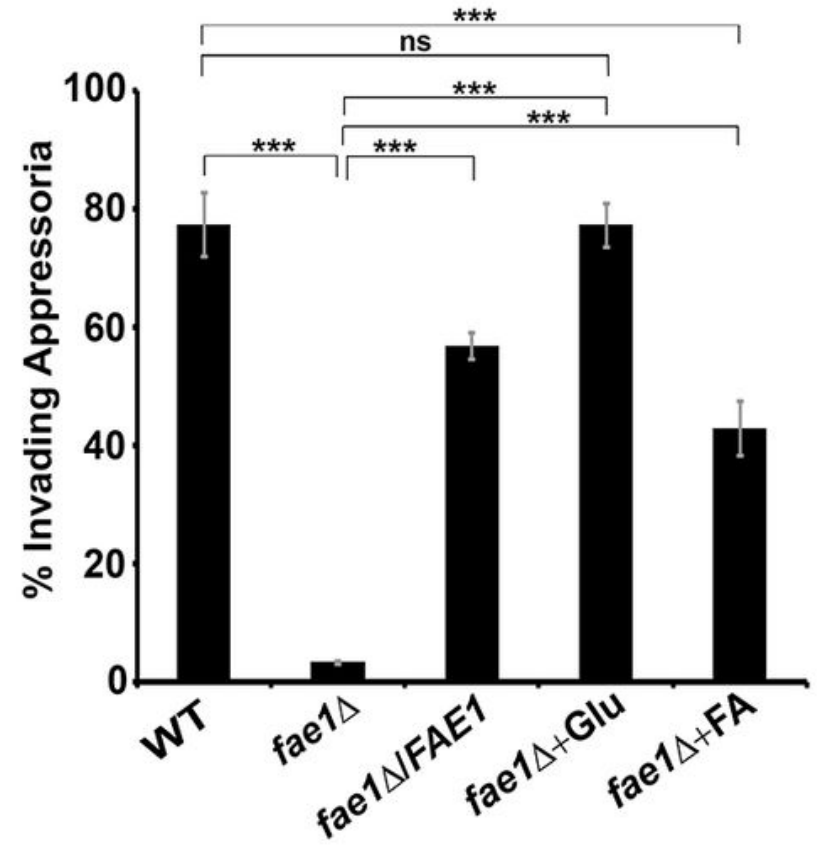

B

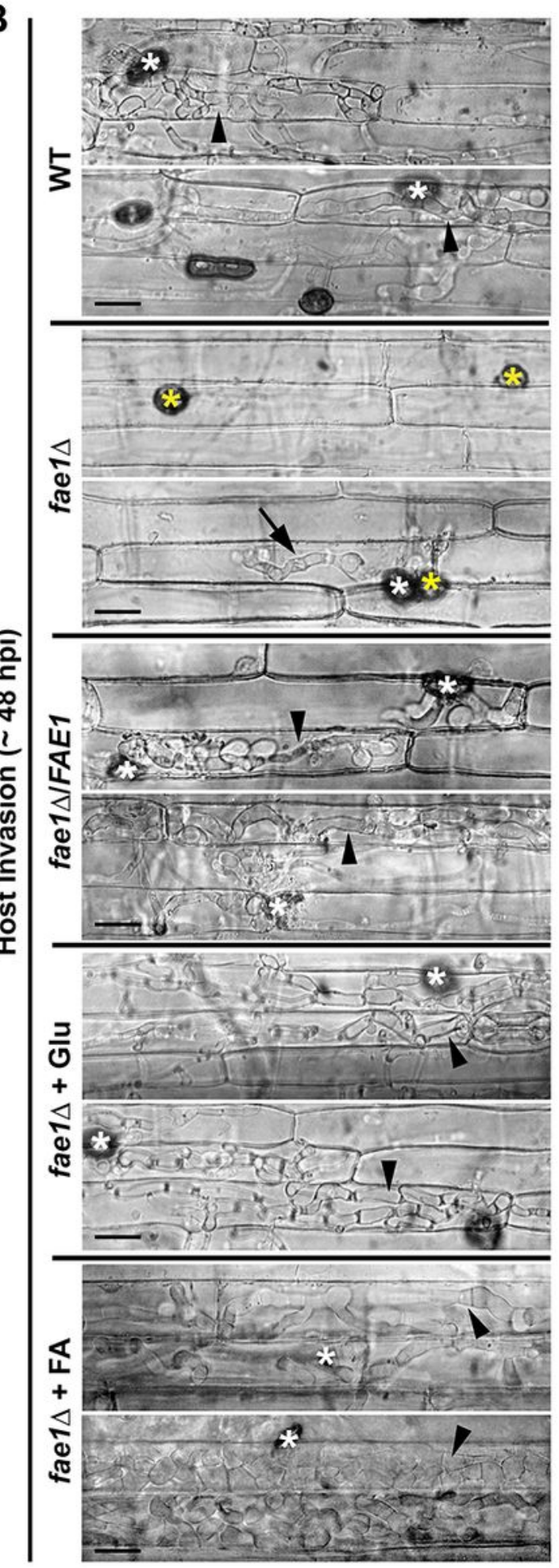


Figure 5

A

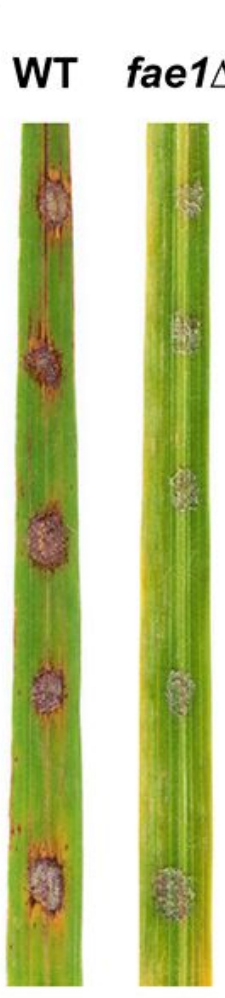

fae1 $\Delta$ fae1 $\Delta$

$+\mathrm{FA}+\mathrm{GSH}$
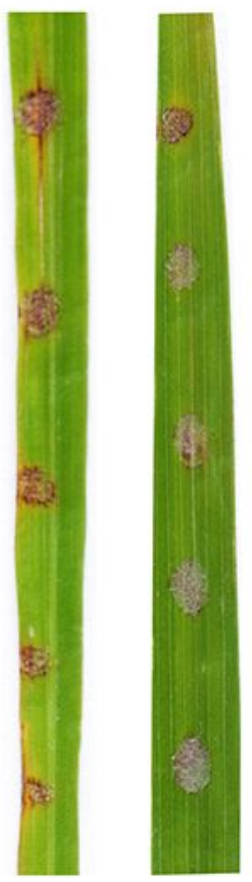

C

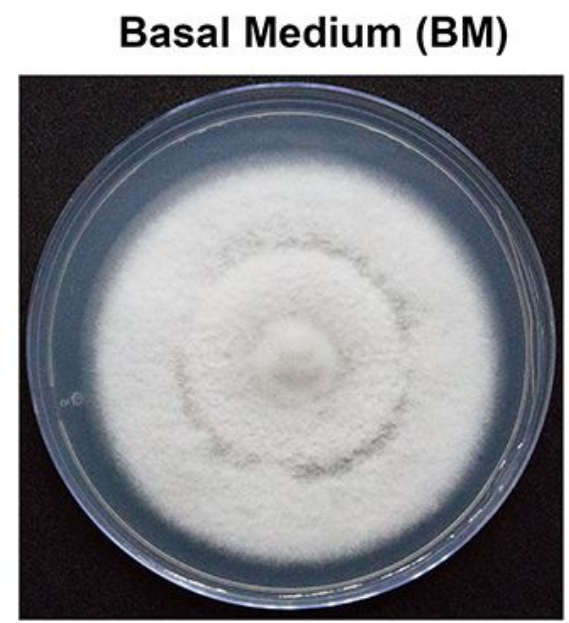

B
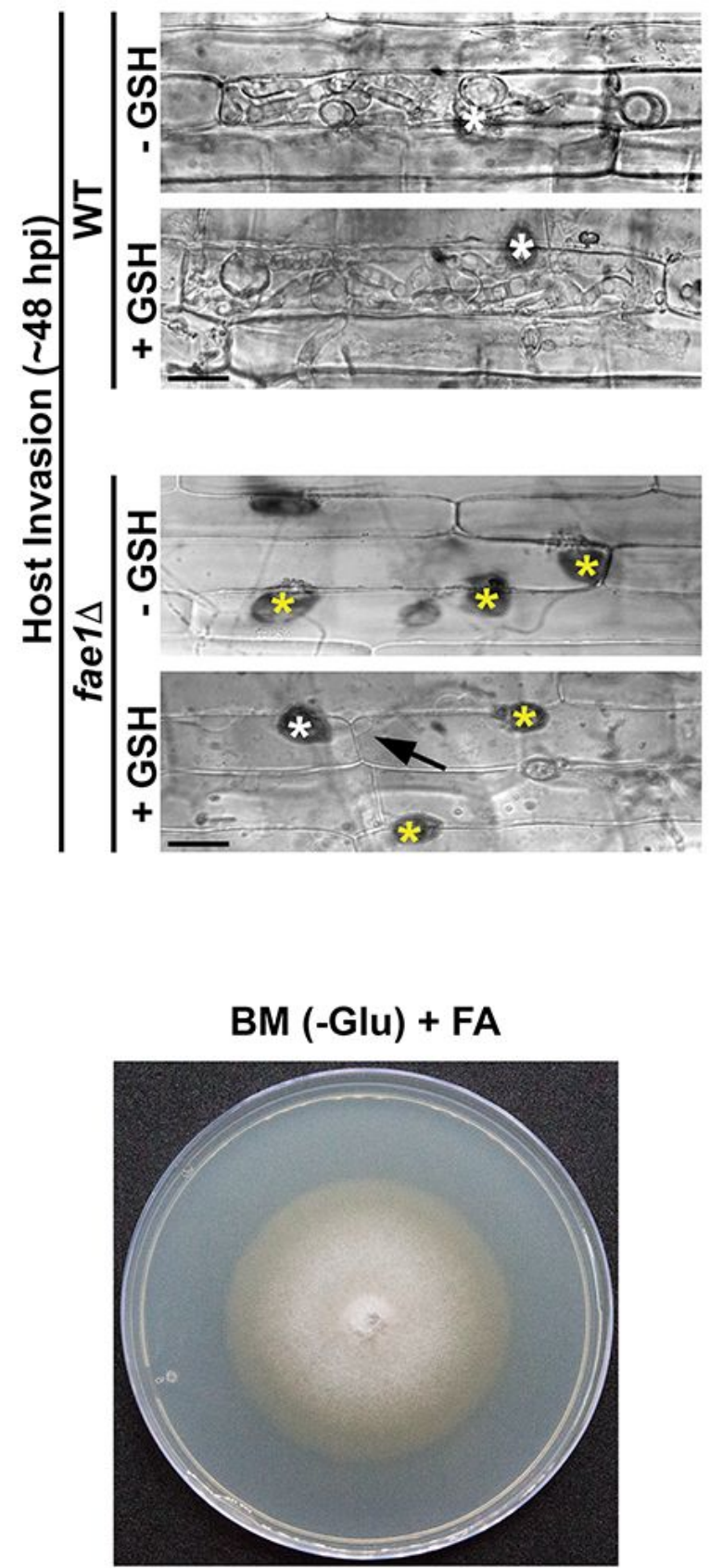

Figure 6

Ferulic acid released by the Fae1 action is likely required as a nutrient source during host invasion and colonisation by $\mathrm{M}$. oryzae. (A) Drop-inoculation assay showing infection ability (on detached rice leaves) of WT, fae $1 \Delta$ or fae $1 \Delta$ supplemented with $100 \mathrm{mM}$ ferulic acid (FA) or $20 \mathrm{mM}$ reduced glutathione (GSH). 
Images were taken at $6 \mathrm{dpi}$. (B) Leaf sheath inoculation assay showing host invasion ability of the WT or fae $1 \Delta$ supplemented with $20 \mathrm{mM} \mathrm{GSH}$, which was added at $22 \mathrm{hpi}$. The results were observed at $48 \mathrm{hpi}$. White and yellow asterisks mark the invading and non-invading appressoria, respectively. Arrow depicts invasive hypha restricted to the first host cell invaded. Scale bar, $10 \mu \mathrm{m}$. (C) Vegetative growth of the WT M. oryzae on basal medium (BM) with or without $0.01 \%$ ferulic acid. The images were taken at $10 \mathrm{dpi}$.

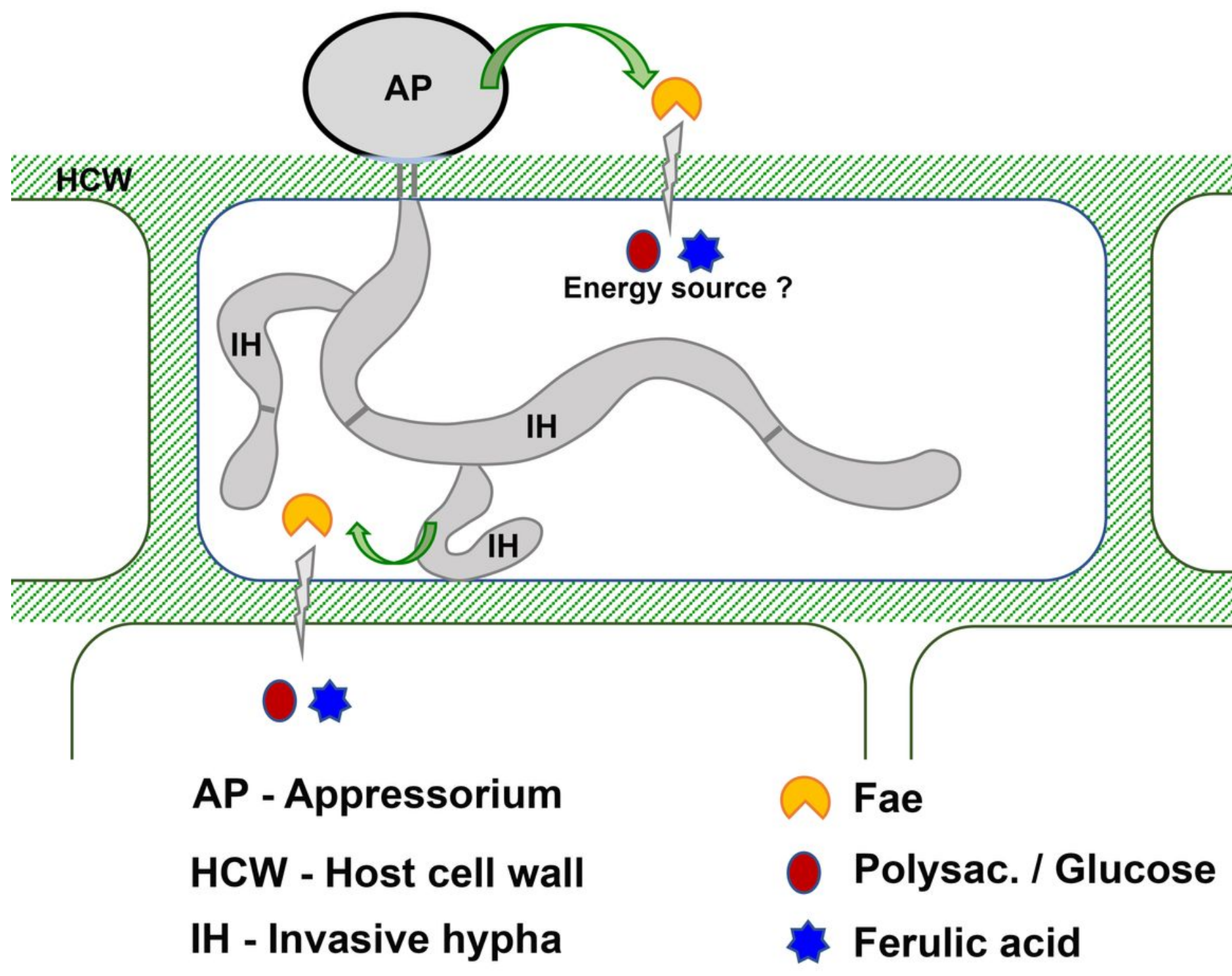

Figure 7

A proposed model of Fae1 function during pathogenesis in M. oryzae. Fae1, likely secreted along with other CWDEs, hydrolyses the plant cell wall to release ferulic acid and constituent carbohydrates during penetration of the first host cell and subsequent spread to the neighbouring cells. Released ferulic acid, the product of Fae enzyme action, and/or glucose, the breakdown product of cellulose, likely act as an energy source enabling successful host-invasion and colonisation by the blast fungal pathogen. 


\section{Supplementary Files}

This is a list of supplementary files associated with this preprint. Click to download.

- SupplementaryInformationThakeretal.pdf 